Ann. Zootech., I968, 17 (2), Io7-I42.

\section{I.N.R.A. \\ BIBLIOTHEQUE UO 35906 \\ Domaine de CROUELLE}

63039

Clermont-FD CeDEX 2

\title{
ÉTUDE DE QUELQUES FACTEURS INFLUENÇANT LES DIFFIGULTÉS DE VÊLAGE EN CROISEMENT INDUSTRIEL
}

\author{
M. BELIC ( ${ }^{1}$ ) et F. MENISSIER \\ Station centrale de Génítique animale, \\ Centre national de Recherches zootechniques, 78 -Jouy-en-Josas \\ Institut national de la Recherche agronomique
}

SOMMAIRE

Ine brève analyse de l'origine et de la nature des difficultés de vêlage, nous a permis de dégager les principaux facteurs responsables des vêluges dystocirpues dans le cas du croisement de première génération.

Notre étude persomelle a porté sur les données de contrôle de descendance de taureaux Charolais et Limousins utilisés en race pure et en croisement de première génération, durant la période : $1960-65$ ( 7500 vêlages environ).

A partir de ces données, nous avons mis en évidence l'influence du poids de naissance du veau, de son sexe, du format de sa mère, des races maternelle et paternelle, sur les conditions de déroulement du vêlage ; par contre, l'incidence du caractère "culard " chez le père, n a pas semblé modifier l'importance des difficultés de vêlage, lors des accouplements avec des femelles adultes de type "normal".

Nous avons estimé l'héritabilité du taux de vêlages difficiles, it $: h^{2}=0,05$. Le coefficient de corrélation génétique (entre taureaux) reliant le taux de vêlages difficiles et le poids moyen des veaux à la naissance, est positif et très voisin de l'unité.

\section{I. - OBJE'T DE L'ÉTUDE}

Depuis quelques décennies le consommateur demande de plus en plus une viande bovine tendre, maigre et à cuisson rapide. L'orientation nouvelle du marché de la viande qui en résulte, a entraîné une modification des types de production et des objectifs de la sélection des bovins à viande. Les animaux sont abattus de plus en plus jeunes et nous sommes conduits, de ce fait, à rechercher ceux présentant une

(1) Adresse actuelle : Institut zootechnique, Svetog Save 43, Belgrade (Yougoslavie). 
vitesse de croissance élevée pendant la première année (production de taurillons) voire les premières semaines de vie (production de veaux). Or l'existence de coefficients d'héritabilité plus élevés pour le poids à la naissance que pour la croissance durant les deux ou trois premiers mois d'une part, de corrélations génétiques également élevées entre ces deux critères d'autre part (POUJARDiEU et Vissac, I968), risque d'entraîner indirectement une augmentation de la croissance du foetus in utero.

En outre, l'amélioration génétique de la croissance musculaire des jeunes bovins s'obtient essentiellement par des techniques de croisement à but commercial : croisement industriel, croisement d'absorption. Ces croisements comportent en général l'accouplement de mâles à forte croissance musculaire (Charolais par exemple), avec des femelles de taille ou de compacité plus réduite. Ces femelles appartiennent soit à des races laitières (nord de la France et de 1'Europe) soit à des races à fins multiples qui ne sont plus soumises à la traite (centre et sud de la France), soit à des races spécialisées pour la production de viande et dont 1'adiposité est trop accusée (races à viande Anglo-saxonnes en Argentine). Les accouplements ainsi réalisés conduisent parfois à un déséquilibre morphologique qui se manifeste, au moment de la parturition, par des difficultés de vêlage.

La nature et l'importance de ces difficultés varient énormément. Eilles sont signalées par de nombreux auteurs relatant les résultats d'essais de croisements avec des taureaux Charolais entre autres (LOPEZ S.AUBIDET et al., I963; Veterinary clinical observation unit, Ig63; REYNEKE et PENZHORN, I964; TURTON, I964; CROWIEY, I965; BOYAZOGI,U et HARWIN, I966; EdwARIS et al., I966; HANSEN, I966 ; LINDHE, Ig66).

L'incidence des difficultés de vêlage sur la rentabilité des spéculations bovines peut ne pas être négligeable. Dans les exploitations de petite taille, elles exigent une plus grande surveillance du cheptel pendant la période des mises bas, ainsi que le recours plus ou moins fréquent à l'opération " césarienne ". Cette pratique s'accompagne d'un taux de mortalité des veaux ou des mères plus élevé et, souvent, d'une infécondité passagère ou permanente des femelles : en Belgique, l'allongement de l'intervalle entre vêlages, consécutif à l'opération césarienne dépasse parfois un mois (HANSET, I966). Dans le cas de races à viande dont les vêlages sont saisonniers (charolaise), les conséquences économiques de cette infécondité sont encore plus graves puisqu'elle risque de repousser le vêlage suivant " hors-saison». Iin ce qui concerne le taux de mortalité des veaux issus de vêlages difficiles, les résultats de Hanswn (I966) relatifs à des croisements $q$ Jersey $\times \hat{o}$ Charolais sont particulièrement spectaculaires :

- vêlages faciles $=\ldots \ldots 86,7$ p. Ioo (taux de mortalité $=3$, I p. Ioo)

- vêlages "forcés " $=\ldots \ldots 6,6,8 \mathrm{p}$. Ioo (taux de mortalité $=$ I2,o p. Ioo)

- vêlages avec intervention vétérinaire $=6,5 \mathrm{p}$. Ioo (taux de mortalité $=34,6 \mathrm{p}$. I00)

Dans le cas des troupeaux à grands effectifs et élevés de façon extensive, où la surveillance du cheptel est de ce fait plus difficile, les cas de mortalité de la mère, du veau ou du couple peuvent évidemment être beaucoup plus fréquents. Sur le plan de l'élevage, il importe en définitive, comme le font remarquer GrunsELL et al. (r963), de chiffrer les conséquences économiques de ces troubles de parturition dans le cas du croisement et de les comparer à l'excédent de recettes fourni par les veaux croisés. 
Cette étude est consacrée à l'analyse des causes de variation des difficultés de vêlage sur les populations de femelles de races variées soumises au croisement de première génération avec des taureaux Charolais ou Blonds du Sud-Ouest en vue de la production de veaux de boucherie (Centre et Sud de la France).

\section{2. - NATURE ET ORIGINE DES DIFFICULTÉS DE VÊLAGE}

Après avoir effectué une description rapide des différentes phases du vêlage, nous présenterons une analyse schématique des facteurs, d'origine génétique ou non, susceptibles d'entraîner des troubles de parturition dans le cas d'une incompatibilité morphologique foeto-maternelle.

\section{I. Description du vêlage et nature des difficultés de parturition}

Le vêlage groupe " un ensemble de faits physiologiques qui aboutissent à la naissance du produit, puis à l'expulsion du placenta et des enveloppes annexielles » (DÉRIVAUX, I957). Il comprend une série de phénomènes qui s'organisent en trois phases successives (DÉRIVAuX, I957; CLEGG, I959).

- Une phase de préparation : elle débute quelques jours avant le part proprement dit et se caractérise par un commencement de dilatation du col, ainsi que par les premières contractions rythmiques de la paroi utérine (C $C_{\text {EGG }}$, I959). Plusieurs signes externes permettent de reconnaître l'approche du vêlage : mamelle congestionnée, lèvres de la vulve légèrement gonflées et tuméfiées, écoulement des produits de lyse du bouchon muqueux du col, affaissement des ligaments sacro-sciatiques. En somme, la vache prépare le passage du fotus en augmentant l'ouverture de son canal pelvien. Au cours de cette phase, se trouvent déterminées la présentation et la position du fœtus.

- Une phase d'expulsion : elle comprend d'abord l'engagement du fotus, puis son acheminement au travers du canal pelvien complètement dilaté. Diverses modifications, dont la mobilité đes articulations sacro-iliaques et l'élargissement des diamètres du bassin, permettent à la " filière pelvienne » d'atteindre ses dimensions maxima (DÉRIVAux, I957). Cette phase exige, de la part de la vache, des efforts expulsifs considérables; quant au veau, il subit des compressions, essentiellement thoraciques, lors de son passage forcé au travers du canal pelvien.

- La phase finale de la mise bas consiste dans le détachement et l'expulsion des enveloppes fœetales et intéresse la mère seule ; elle peut être influencée toutefois par les conditions de l'accouchement.

Si nous exceptons, en définitive, les cas de présentation ou de position anormale du fœetus, les difficultés de vêlage concernent en général le franchissement du canal pelvien par le foetus. La résistance à ce franchissement est fonction đu rapport existant entre le diamètre de ce canal pelvien dilaté et celui du fœetus au niveau du thorax ou des hanches. FAGOT (I964) attribue à une anomalie de ce rapport 9o p. Ioo des opérations césariennes. Il distingue l'excès de volume absolu du veau, de l'insuffisance de dilatation du canal pelvien qui seraient à incriminer dans respectivement 60 p. Ioo et 30 p. Ioo des cas. VANDEPLASSche et al. (I965), VANDENBUSSCHE et al. 
(I964), FRTEDLI (I966) considèrent également l'excès de volume du fotus comme cause essentielle des vêlages difficiles.

L'appréciation des difficultés de vêlage, dans le cadre des enquêtes à grande échelle réalisées dans un but de sélection, reste en général sommaire. Les questionnaires établis à l'usage des techniciens et plus souvent des éleveurs, distinguent, suivant les auteurs 2, 4 ou 5 catégories de vêlages. Cette classification est basée sur l'assistance ou la non-assistance de l'éleveur, sur la présence ou l'absence du vétérinaire. Ainsi BONFERT (I964), REYNEKE et PENZHORN (I964), HANSEN (I966) considèrent 3 catégories :

$\mathrm{I}=$ vêlages faciles sans assistance,

$2=$ vêlages avec assistance de l'éleveur,

$3=$ vêlages nécessitant l'intervention du vétérinaire.

Certains auteurs ont essayé de mieux caractériser l'importance de l'assistance fournie par l'éleveur à la femelle, par l'indication du nombre maximum de personnes assurant une traction sur le veau lors de la parturition : I, 2, 3, ou plus (CrowLEY, I965). Parfois aussi, la nature des difficultés de vêlage et surtout de l'intervention pratiquée par le vétérinaire, est précisée (AURIoL et al., I96I). Enfin plusieurs auteurs considèrent les conséquences de ces difficultés quant à la viabilité des produits (DONAlid, I963; VAN DIETEN, I963; CROWLEY, I965 ; VANDEPLASSChE et al., I965 HANSEN, I966; HaNset, I966). Toutes ces modalités varient donc, en définitive, suivant les conditions de réalisation des enquêtes.

\section{2. Facteurs responsables des difficultés de vêlage liées à une incompatibilité morphologique focto-maternelle.}

\section{2. I. Facteurs régissant l'amplitude du canal pelvien de la femelle.}

Cette amplitude dépend, d'une part, des diamètres normaux du canal pelvien de la vache, fonction de son anatomie, d'autre part, de l'intensité des phénomènes physiologiques de dilatation du bassin, lors de la parturition.

Les variations morphologiques du bassin et du canal pelvien sont liées à des influences génétiques et nutritionnelles intervenant sur le format, la conformation ou l'état d'embonpoint de la femelle (Boyd et HAFs, r965). L'âge de cette dernière, ainsi que le nombre de ses vêlages antérieurs, influencent également son anatomie et les dimensions de la filière pelvienne (BEN DAviD, I964). Nous savons à ce sujet, que l'angustie pelvienne se rencontre fréquemment chez les génisses à la suite d'un vêlage trop précoce. Il convient, en effet, de rappeler que les segments osseux proximaux des membres et le bassin figurent parmi les parties du squelette dont le développement est le plus tardif (Hammond, I955). Si la soudure des trois pièces osseuses limitant le canal pelvien (ilium, ischium, pubis) se réalise vers 7-Io mois, l'ossification de certaines épiphyses de l'ilium et de l'ischium (noyaux secondaires) n'interviendrait définitivement que vers 5 ans (BARONE, I966). De plus, les femelles primipares présentent, au niveau de la symphyse ischio-pubienne, une crête (tubercule pubien dorsal) qui, non seulement réduit l'ouverture du détroit antérieur, mais gêne l'expulsion du fœtus, puisqu'elle peut dépasser $5 \mathrm{~cm}$ de hauteur (DEGHILAGE, I9II, cité par FAGOT, I964). En ce qui concerne les variations des dimensions du bassin 
et $d u$ tractus génital des femelles de même âge, nous savons qu'elles peuvent être liées, en partie, à des différences génétiques entre races ou intra-races, ainsi qu'à des influences nutritionnelles sur la croissance globale de la génisse (SwANson et HrN'ron, I964). Quant à la conformation spécifique du bassin d'animaux de même taille, FAGOT (I964) et DÉRrvaux et al. (Ig64) notent, chez certaines vaches à fort développement musculaire, une déformation des coxaux entraînant un rétrécissement du détroit antérieur et conduisant à des vêlages difficiles. Par ailleurs, il a été montré (VISSAC, Ig62) que 1'hypertrophie musculaire d'origine génétique (culard) s'accompagnait d'une réduction particulièrement nette des dimensions du coxal. L'état d'embonpoint de la femelle, lié à son régime alimentaire en fin de gestation, peut également gêner le passage du fœetus et provoquer des parturitions difficiles (LOPEZ SAUBidet et al., Ig63; BALIKA, I965).

Les variations de dilatation du canal pelvien, lors de la préparation au vêlage (première phase), et de contraction de 1'utérus sont probablement à relier à la surcharge de la matrice et, surtout, à un dysfonctionnement hypophysaire de l'individu (DÉrIVaux, I957). Nous savons à ce sujet qu'il se manifeste des différences entre les génisses et les vaches adultes, ainsi qu'entre types génétiques diffétents : le manque de préparation des femelles culardes au moment du vêlage a été signalé notamment par Ropo' (I967).

\section{2. 2. Facteurs intervenant sur la taille et la conformation du fatus.}

Ces facteurs sont, soit d'origine génétique, soit liés à l'influence utérine, fonction elle-même du génotype de la femelle et du milieu dans lequel elle vit. Ils peuvent agir les uns et les autres aussi bien sur la durée de gestation étroitement liée au poids du veau à la naissance, que sur la croissance proprement dite du foetus in utero.

En ce qui concerne l'incidence du génotype du veau (paternel et maternel), elle se manifeste d'abord par l'intermédiaire de son sexe; les mâles étant plus lourds, plus compacts et portés plus longtemps par la femelle. On sait par ailleurs que ce génotype et notamment la fraction transmise par le père, intervient directement sur la durée de gestation du veau (JAFAR et al., I950). Ennfin les variations de poids à la naissance des veaux entre races paternelles ou entre descendances de taureaux sont notables puisque le coefficient d'héritabilité de ce poids est souvent supérieur à celui de la croissance post-natale (PoujARDiEu et ViSSAC, I968). En dehors de l'effet utérin proprement dit, le génotype de la femelle intervient enfin, au même titre que celui du mâle, sur la durée de gestation, le poids et la conformation à la naissance du veau.

L'importance de l'effet maternel utérin sur ces deux derniers critères a été montré par JouBERT et HAMmond (I958) à la suite d'accouplement réciproques de bovins appartenant à des races de tailles très différentes. L'effet maternel sur le poids du fotus peut aussi être déterminé par l'action du milieu, et notamment du niveau nutritionnel, que ce soit au cours de la croissance de la génisse, que ce soit en fin de gestation. Dans le premier cas, un régime restreint interviendrait en diminuant le format adulte de la femelle, sa durée de gestation et le poids de naissance de ses veaux. SigNoRET et al., (I956) ont ainsi trouvé que la durée de gestation des vaches normandes était plus faible dans la zone d'extension de cette race où la taille des femelles est plus réduite, qu'en Normandie. Les effets d'une réduction énergétique de la ration 
en fin de gestation sur le poids de naissance des veaux et la fréquence des difficultés de vêlage, ont été montrés par LoPez SAubideí et al. (I963) et CABrini et CavanDOL, (I 664). Enfin, le mode d'élevage et l'exercice des femelles entraînant une diminution de la durée de gestation (STOCKKLAUSNER, I955 ; SchMidT, I953), peuvent expliquer des différences de poids à la naissance en relation notamment avec la saison.

L'analyse des difficultés de vêlage suivant les phénomènes anatomiques et physiologiques qui les provoquent, suivant les facteurs génétiques ou non qui en sont responsables, reste en définitive très complexe. Nous avons schématisé les différents aspects de cette analyse sur la figure $I$.

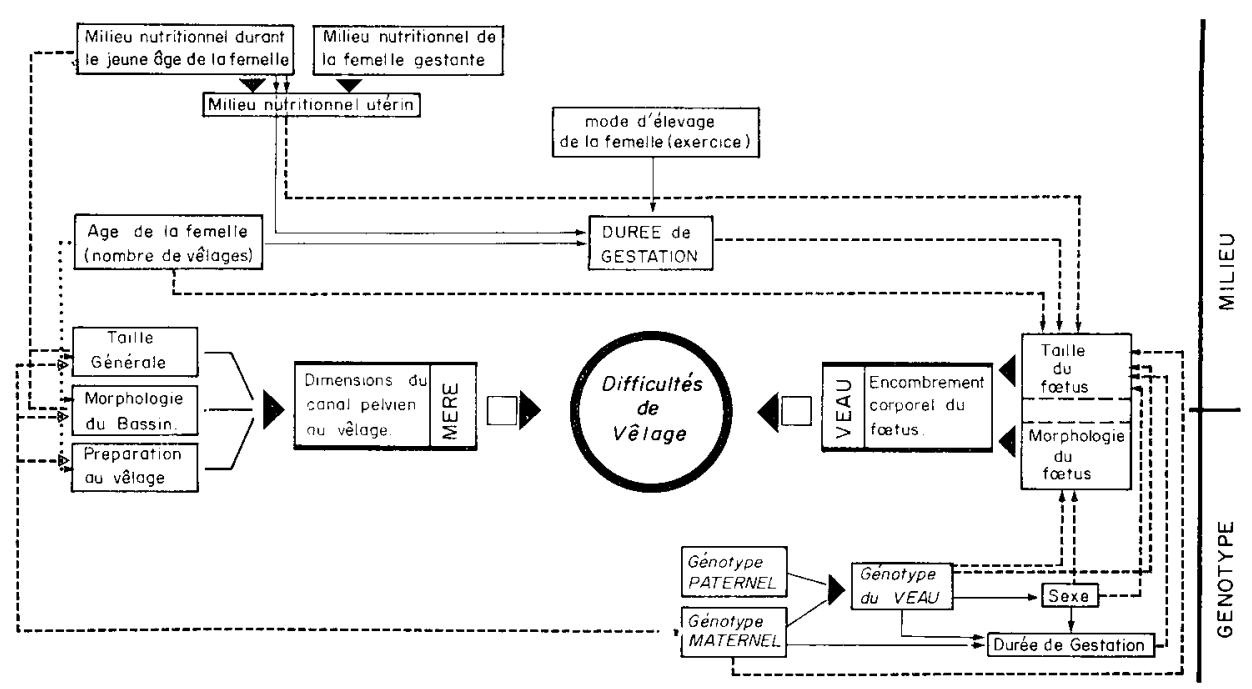

FIG. I. - Nature et origine possibles des difficultés de vêlage (présentation normale)

\section{3. — MATÉRIEL ANIMAL E'T MÉTHODE D'ÉTUDE}

\section{I. Matériel animal}

\section{I. I. Renseignements collectés.}

Cette étude porte sur les données de contrôle de la descendance des taureaux de races à viande françaises en race pure ou en croisement de première génération (période de 1960 à 1965 ) en vue de la production de veaux de boucherie. Les opérations de contrôle de descendance étaient effectuées en général chaque année et pour chaque centre d'insémination artificielle sur une série de taureaux mis simultanément en comparaison. Les renseignements concernant les conditions de vêlage étaient fournis par les éleveurs sur une déclaration de naissance codifiée de la façon suivante :

$\mathrm{I}=$ vêlage facile, sans assistance;

2 = vêlage avec assistance de l'éleveur;

$3=$ vêlage difficile ;

4 = vêlage dystocique, nécessitant une intervention du vétérinaire.

La nature des difficultés rencontrées : présentations anormales, excès de volume du veau, conformations particulières, etc... n'était pas précisée. Par ailleurs, ces renseignements n'étaient recueillis que pour les veaux vivants. L'étude exclut de ce fait les cas d'avortement, de mortinatalité ou de mortalité périnatale. D'autre part, les indications fournies aux éleveurs par les centres d'insémination artificielle, en vue de l'application de la codification ci-dessus, ont pu varier sensiblement d'un centre à l'autre. Ainsi les renseignements recueillis dans le Tarn et l'Aveyron diffèrent notamment de ceux des autres centres. 
Outre les difficultés de vêlage, l'éleveur fournissait également le poids à la naissance du veau. La croissance de ce dernier était contrôlée à partir de pesées périodiques mensuelles et estimée par le poids à 75 jours (intrapolation linéaire), cet âge correspondant à l'abattage des premiers veaux. L'âge de la mère n'était pas enregistré systématiquement et, en général, n'a pas été considéré dans cette étude. Par contre, le format de cette dernière était apprécié par la mesure du tour de poitrine. La conformation du veau était estimée subjectivement suivant une table de pointage définie par VISSAC et al. ( ( 965$)$. Enfin, les races parentales et l'identité du père ainsi que le sexe du veau étaient connus. Nous n'avons conservé dans cette étude que les données relatives à des mères présumées de race pure.

\section{I. 2. Échantillons considérés et schémas d'observation.}

Étude des différences entre races maternelles, taureaux et sexes des z'eaux.

Cette étude a porté sur les séries de taureatix soumis simultanément au contrôle de descendance et comportant un effectif suffisant d'animaux. Nous avons regroupé ces séries par centre d'insémination pour tenir compte des variations qui interviennent dans les bases d'appréciation des difficultés de vêlage, d'un centre à l'autre. Les inséminations correspondant aux différentes séries de testage d'un même centre étaient par ailleurs réalisées dans une même zone. Ce sont ces séries regrou-

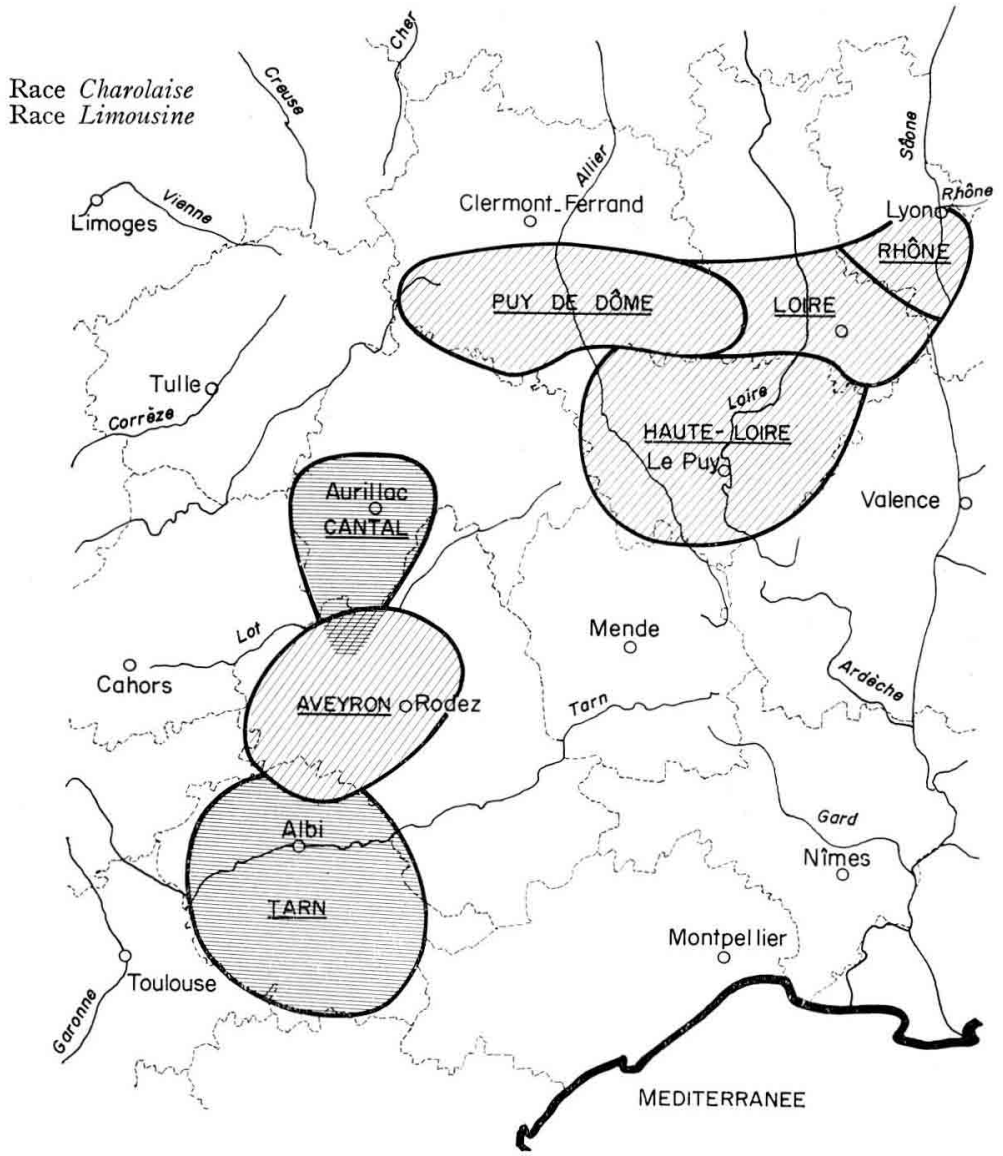

Fig. 2. - Répartilion des zones de lestage considéries suivani les races paternelles

pées que nous avons réparties sur le tableau I par centre et par race de mère (échantillon $n^{\circ} \mathrm{I}$ ). A titre indicatif nous avons fait figurer sur ce même tableau le poids moyen des femelles de ces races tel qu'il ressort des contrôles effectués aux Concours généraux agricoles de Paris (1952-1954). Les régions intéressées et les zones d'extension des races étudiées figurent par ailleurs sur la carte (fig. 2). 
M. BELIC, F. MENISSIER

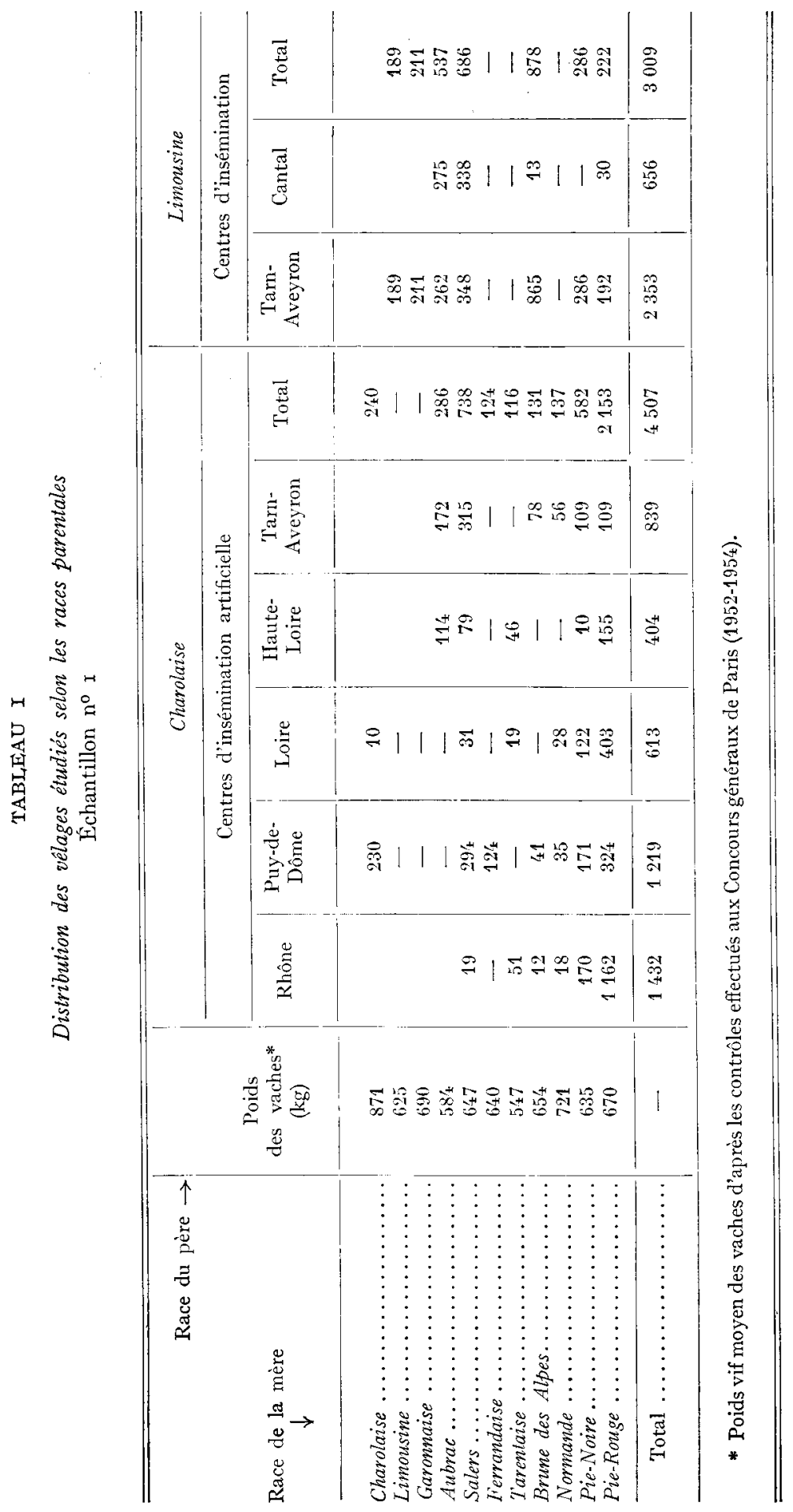


Nous pouvons considérer que le sperme de chaque taureau a été réparti au hasard suivant la race et le format des femelles puisqu'il s'agissait de taurillons de testage dont les performances étaient inconnues des éleveurs et des inséminateurs. 1)ans les comparaisons cntre descendances de taureaux, nous n'avons en fait considéré que les séries de veaux issus de taureaux Charolais, les autres races à viande n'étaient pas suffisamment représentées pour donner lieu à une analyse statistique.

Étude des différences entre races paternclles et types de taureaux.

La comparaison des difficultés de vêlage liées à la race du père ou à certains génotypes (caractère culard) n'était pas possible, ¿̀ partir de l'information ci-dessus. Iin effet, dans la mesure où plusieurs races ou types de taureaux peuvent être utilisés dans une même région, l'éleveur tend à orienter l'emploi du taureau Charolais, surtout s'il présente le caractère culard, vers les femelles adultes et de grande taille.

J'incidence de ces facteurs a donc été étudiée séparément a l'occasion d'expériences spécialement planifiées dans ce but. En ce qui conceme l'influence de la race paternelle, nous avons considéré un échantillon (échantillon $n^{\circ}$ ) de veaux issus de taureaux ( 8 pour chacue race) des trois principales races à viande exploitées en France: Charolaise, I inousine et Garonnaise. Ces taureaux ont été utilisés en croisement de première génération sur des femelles de races : Nomande, Pie-Noire et Garonnaise. Le sperme des taureaux des trois races i viande a été réparti au hasard sur les femelles quels que soient leur âge et leur format. En fait, il n'a pas été possible d'éviter un choix de la part de l'éleveur qui s'est souvent refusé à utiliser le taureau Charolais sur ses génisses. Pour cette raison, nous avons cxclu ces femelles primipares de notre étude. J)ans ces conditions le nombre de vêlages enregistrés selon les races paternelle et maternelle, est rapporté clans le tableau 2.

TABLEAU 2

Distribution des vêlages étudiés selon le type de croisement Echantillon $\mathrm{n}_{2}^{\circ}$ - (femelles adultes)

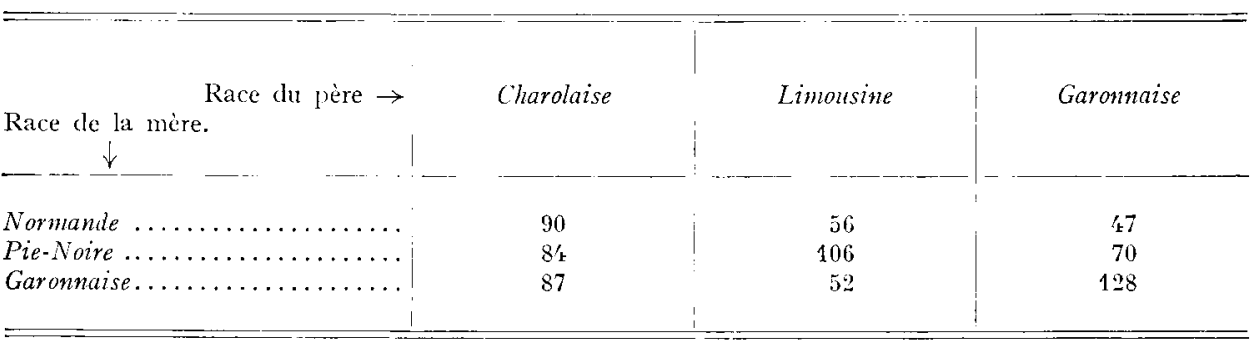

I.e troisième échantillon est constitué de la descendance de taureaux Charolais porteurs du caractère culard et de taureaux normaux (3 de chaque type). Ces taureaux ont été utilisés dans les centres de IIaute-Loire et du Rhône. Le sperme de ces taureaux est également supposé distribué au hasard suivant la race, l'âge et la morphologie (format et développement musculaire) des femelles qui appartiennent à des races rustiques ou laitières. La répartition des observations selon les centres et les phénotypes des taureaux figure sur le tableau 3 .

\section{TABLEAU 3}

Distribution des vêlages étudiés selon le centre d'insémination artificielle et le phénotype des taureaux utilisés

Échantillon $n^{0} 3$ - (femelles adultes)

\begin{tabular}{|c|c|c|}
\hline $\begin{array}{ll} & \text { Centre de } \rightarrow \\
\text { Phénotype } & \\
\text { des taureaux } & \\
\downarrow & \end{array}$ & Ifante-Loire & Rhône \\
\hline $\begin{array}{l}\text { Culards. . . . . } \ldots \ldots \ldots \ldots \ldots \\
\text { Normaux } \ldots \ldots \ldots \ldots \ldots \ldots\end{array}$ & $\begin{array}{l}2 / 1 \\
38 ;\end{array}$ & $\begin{array}{l}213 \\
153\end{array}$ \\
\hline
\end{tabular}




\section{2. Méthodes biomítriques}

Les proportions de vêlages difficiles et dystociques (types : $3+4$ selon la codification ci-dessus) ou de vêlages nécessitant une assistance quelconque (types : $2+3+4$ ), ont été comparées respectivement aux proportions de vêlages faciles (types : $\mathrm{I}+2$ ) ou de vêlages non assistés (types $: I$ ), suivant différents critères et à partir du test de $\chi^{2}$. Les valeurs de $\chi^{2}$ correspondant à un critère donné (sexe par exemple), pour plusieurs sous-échantillons indépendants relatifs à un autre critère (race maternelle par exemple) ont été regroupés suivant la méthode décrite par Simpson et al. (I960). Nous avons par ailleurs caractérisé les différences de difficultés de vêlage entre deux groupes (mâles et femelles par exemple) appartenant à plusieurs échantillons (région ou race), ì partir de la statistique :

$$
\frac{\sum_{i=1}^{i=n} \sqrt{\chi_{i}^{2}}}{\sqrt{n}}
$$

considérée comme une variable centrée réduite $\left(^{1}\right): \chi_{i}^{2}$ est la valeur de $\chi^{2}$ pour le ieme échantillon et $n$ le nombre de degrés de liberté relatif au $\chi^{2}$ total c'est-à-dire le nombre d'échantillons.

Ensuite nous avons effectué une recherche systématique des variables continues les plus liées à l'apparition des difficultés de vêlage, suivant la méthode de sélection progressive décrite par WAGNER (I965). Le critère de sélection retenu était le $\Delta^{2}$ proposé par REyMENT (I962) pour discriminer deux groupes d'individus (vêlages faciles et difficiles dans notre cas) suivant une fonction linéaire de variables continues : $\mathrm{X}_{i}, \mathrm{X}_{j}$. Dans ce but nous avons associé à chaque vêlage difficile ( ${ }^{\mathrm{e}}$ groupe), un vêlage facile ( $2^{\mathbf{e}}$ groupe) tiré au hasard parmi les vêlages relatifs à des veaux de même sexe, nés dans la même région, de femelles de la même race, et issus du même père. Soient $\mathrm{X}_{i}^{\prime} \mathrm{X}_{j}^{\prime}, \mathrm{X}_{i}^{\prime \prime} \mathrm{X}_{j}^{\prime \prime}$, les variables indicées $i$ et $j$ respectivement pour les deux individus $\mathrm{A}^{\prime}$ et $\mathrm{A}^{\prime \prime} \mathrm{d}$ 'un couple ainsi constitué.

En posant $\delta_{i}=\mathrm{X}_{i}^{\prime}-\mathrm{X}_{i}{ }^{\prime \prime} \quad \delta_{j}=\mathrm{X}_{j}^{\prime}-\mathrm{X}_{j}^{\prime \prime}$

la statistique $\Delta^{2}$ est définie par :

$$
\Delta^{2}=\sum_{i=1}^{i=k} \sum_{j=1}^{j=k} \omega^{i j} \cdot \tilde{\delta}_{i} \cdot \delta_{j}
$$

$\left(\omega^{i j}\right.$ est la matrice inverse de la matrice des variances et covariances intra groupe des différences $\delta_{i}$ et $\left.\delta_{j}\right)$.

\section{4. - RÉSULTATS BIOMÉTRIQUES}

A partir de l'ensemble des données représentées par les trois échantillons ci-dessus, nous avons essayé de caractériser les influences respectives du sexe du veau, de sa mère et de son père sur les conditions de déroulement de la parturition dans le cas de croisement industriel.

\section{I. Infuence du sexe du veau}

Nous avons étudié ce facteur à partir des données de l'échantillon $n^{0}$ I pour chaque centre et chaque type de croisement. Les sous groupes comportant moins de 30 vêlages pour une race donnée et dans un centre donné, ont été éliminés.

Le pourcentage de vêlages difficiles et dystociques (types : 3 et 4) est généralement plus important avec les veaux mâles qu'avec les veaux femelles (tabl. 4).

(1) A chaque valeur de $\sqrt{\chi_{i}^{3}}$ nous avons attribué un signe arbitraire $(+$ ou - ) selon le sens de la différence des difficultés de vêlages entre les groupes envisagés. 


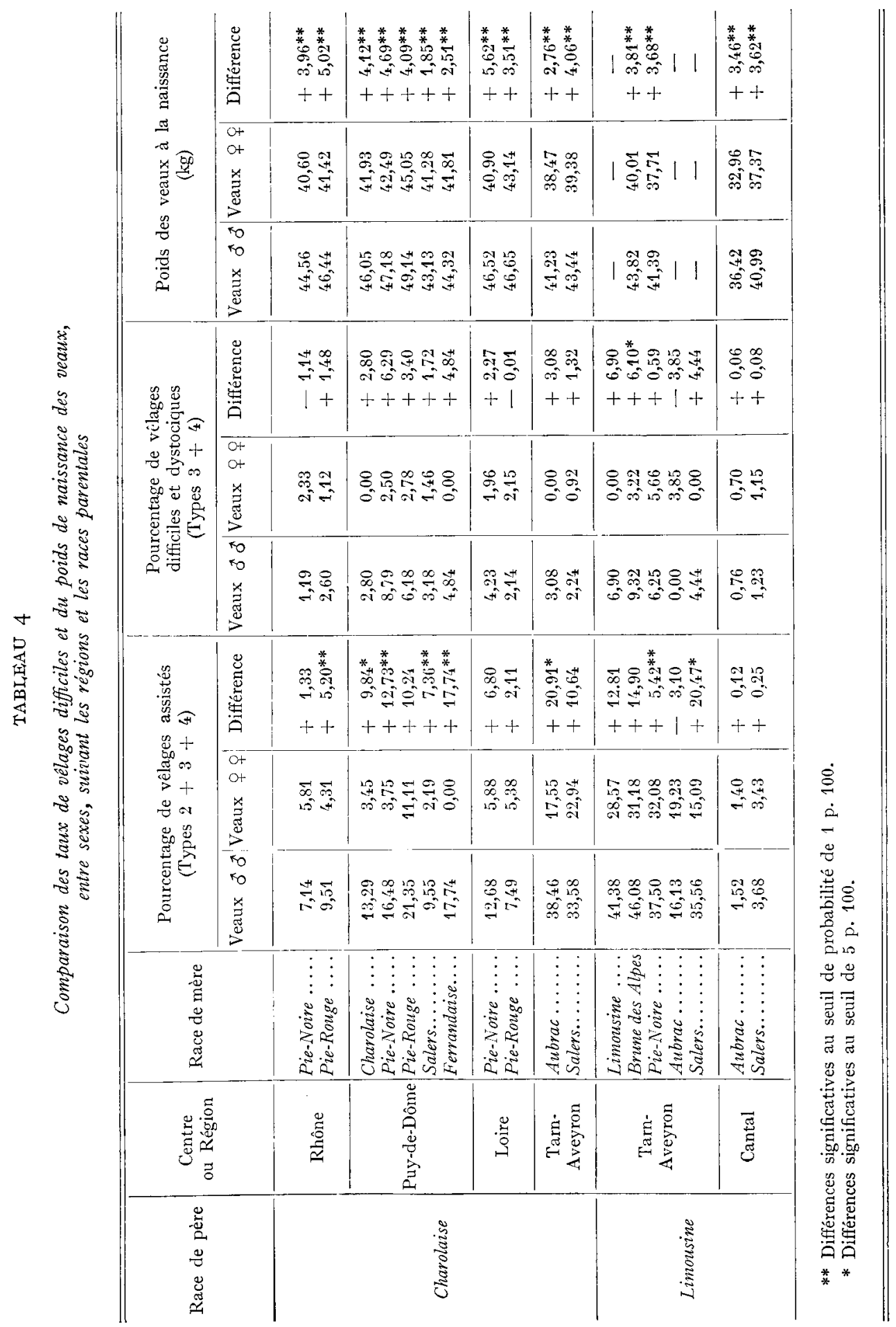


Cependant, les différences de difficultés de vêlages entre sexes sont rarement significatives (tabl. 5). Il n'en va plus de même lorsque nous englobons dans ce pourcentage les vêlages nécessitant une assistance, c'est-à-dire 1'ensemble des types : 2,3 et 4 (tabl. 4 et 6). Cette différence peut en fait être liée à la faible probabilité des vêlages difficiles et dystociques qui, pour un même écart absolu entre les pourcentages relatifs aux mâles et aux femelles correspond à un intervalle de confiance plus large.

L'accroissement des difficultés de vêlage avec les veaux mâles est évidemment à rapprocher de leur supériorité de poids à la naissance par rapport aux femelles (tab1. 4).

\section{2. Influence maternelle}

Sur le même échantillon ( $\left.\mathrm{n}^{0} \mathrm{I}\right)$ nous avons testé statistiquement les différences de difficultés de vêlages entre les races maternelles exploitées dans une même région, et cela pour des veaux de même sexe et issus d'un père de race identique.

Nous avons constaté à nouveau que les différences de fréquence des vêlages assistés entre races (types $2+3+4$ ) sont statistiquement plus nettes que celles relatives aux vêlages difficiles ou dystociques (types $3+4$ ). En général les différences de difficultés de vêlage entre races maternelles ne sont significatives que dans les régions du Puy-de-Dôme et du Tarn-Aveyron, avec des veaux croisés Charolais (tabl. 7 et 8 ). Après regroupement de toutes les valeurs de $\chi^{2}$ indépendamment de la région, l'effet de la race maternelle sur les difficultés de vêlage, est également significatif dans le cas des croisements Charolais, quelle que soit le sexe des veaux. Les valeurs des écarts réduits : $\sum_{i} \sqrt{\chi_{i}^{2}} / \sqrt{n}$ relatifs à l'ensemble de toutes les régions (tabl. 7 et 8 ) font apparaître que les vêlages nécessitant une assistance quelconque (types : $2+3+4$ ) sont plus fréquents dans les races Brune des Alpes, Pie-Noire et Pie-Rouge que dans les races Salers et Aubrac. Par contre, il semble difficile d'établir une hiérarchie entre races maternelles aussi bien à l'intérieur de ces deux groupes, que parmi les autres races (Charolaise, Tarentaise, Ferrandaise, Normande). La signification statistique des effets de la race maternelle ou la " distance" statistique entre ces races (écart-réduit) varie peu selon le sexe des veaux considéré (tabl. 7 et 8 ). Enfin, les variations de poids des veaux à la naissance, entre races maternelles (tabl. 4), peuvent être rapprochées des différences de difficultés de vêlage observées entre ces mêmes races.

Dans un second stade, pour une race de femelle donnée, nous avons cherché à déterminer l'effet du format de la mère, du poids et de la conformation du veau sur les difficultés de vêlage d'après la méthode de sélection progressive des variables décrite ci-dessus. Les écarts intra-couples (vêlages faciles et difficiles) relatifs au poids de naissance des veaux, au format des mères (tour de poitrine), au poids des veaux à 75 jours et à leur prix de vente, ont été utilisés. L,es deux derniers éléments caractérisent respectivement la croissance post-natale et la conformation du veau à la vente. Dans de nombreux cas, le tour de poitrine des mères n'était pas contrôlé; cela nous a conduit à travailler successivement :

- sur les 4 variables énoncées ci-dessus, pour 7 I couples de vêlages,

- sur 3 variables setulement, pour I33 couples de vêlages (le tour de poitrine des mères faisant défaut).

Ensuite, nous avons réalisé la selection progressive des variables dont les fonctions discriminantes des écarts étaient statistiquement les plus différentes de o $\left(\Delta^{2}\right)$ 


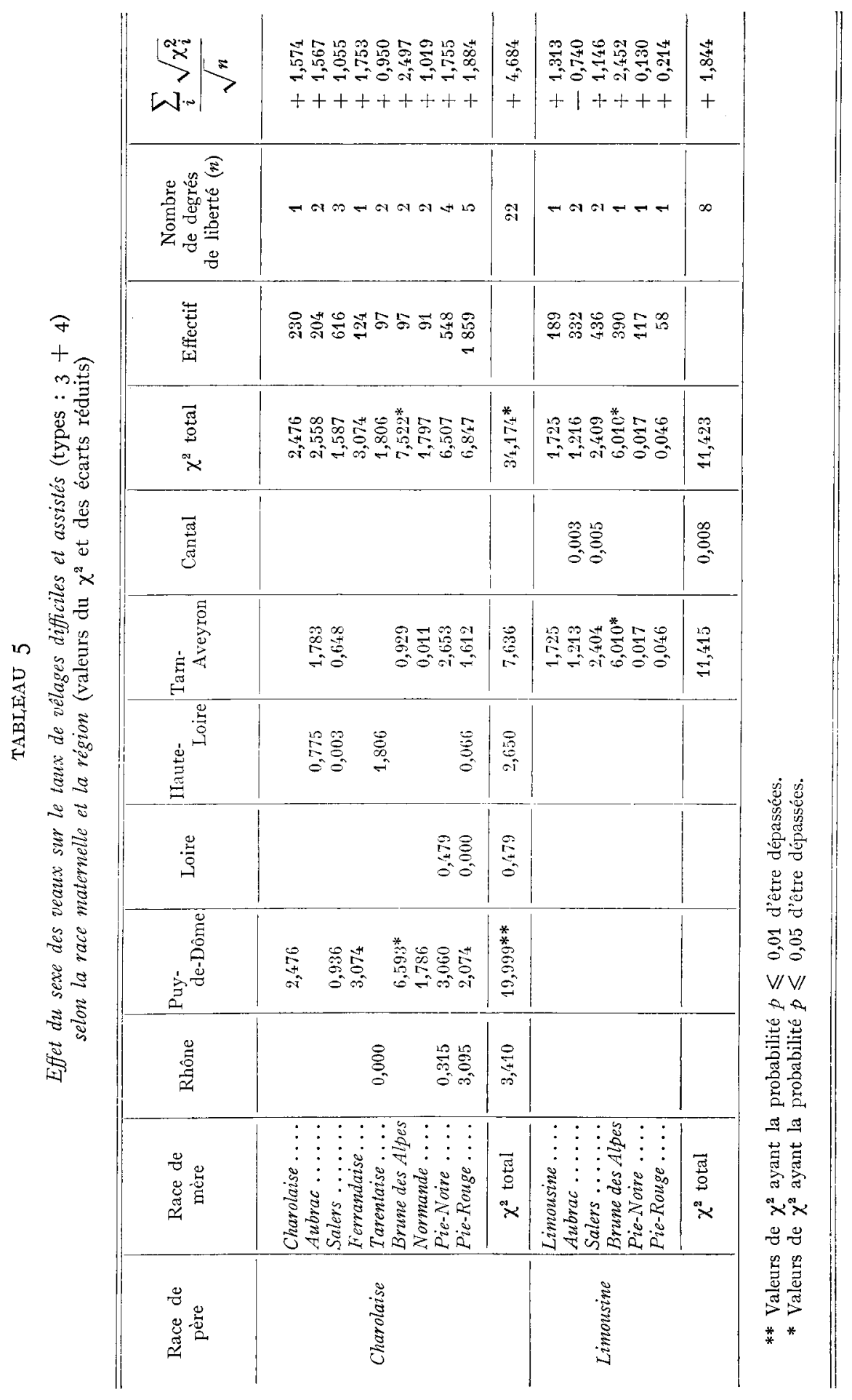




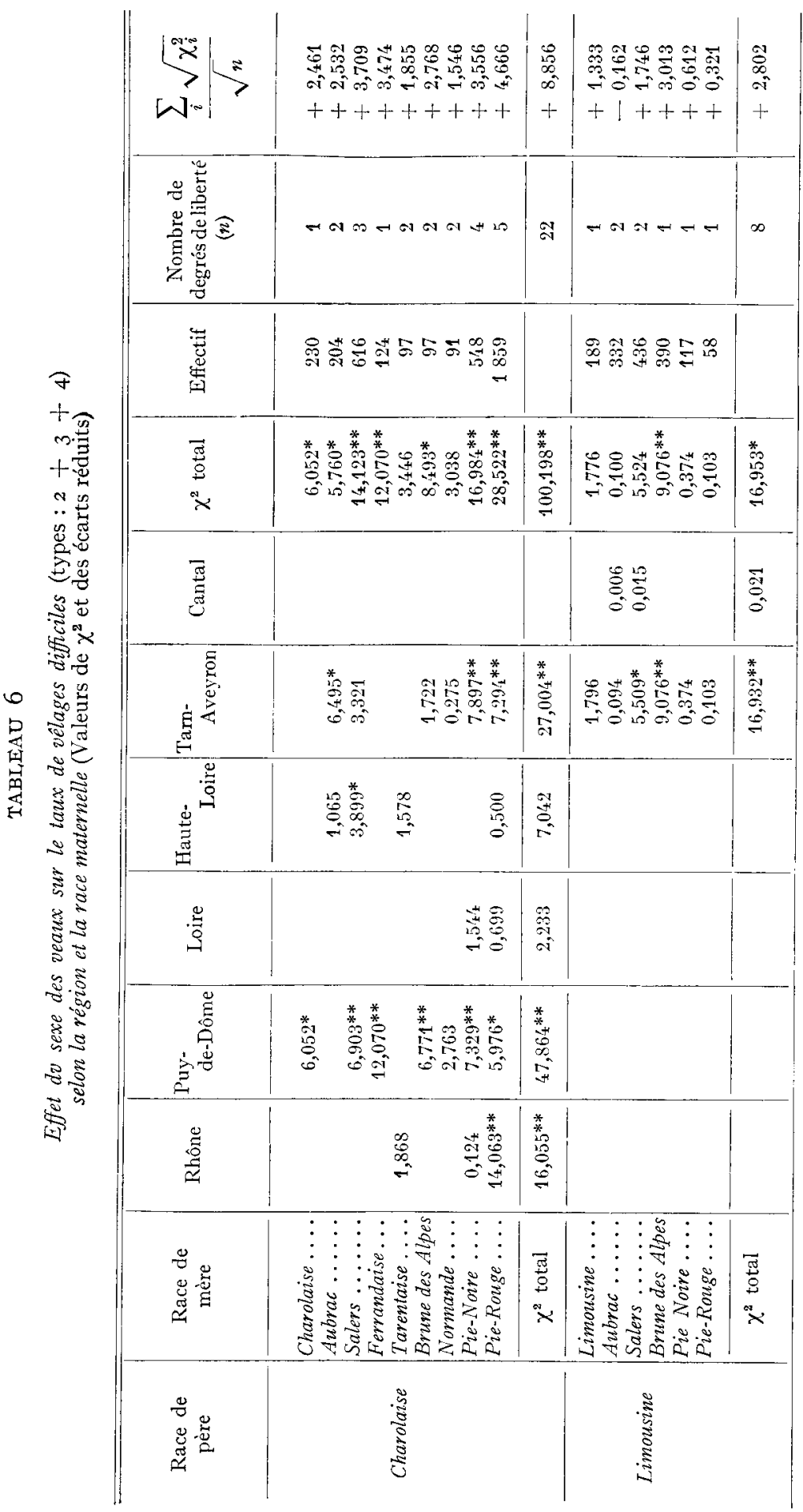




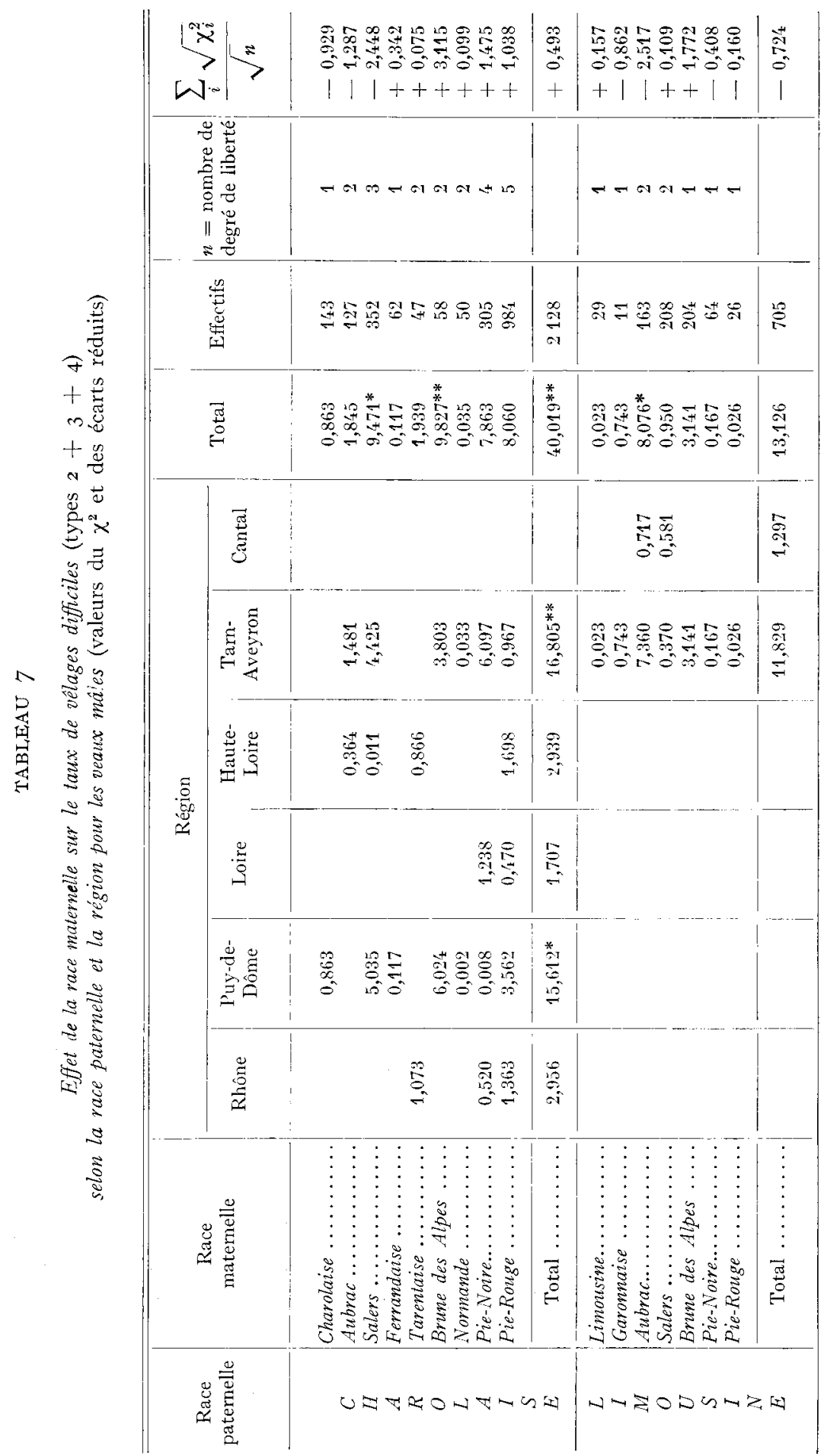




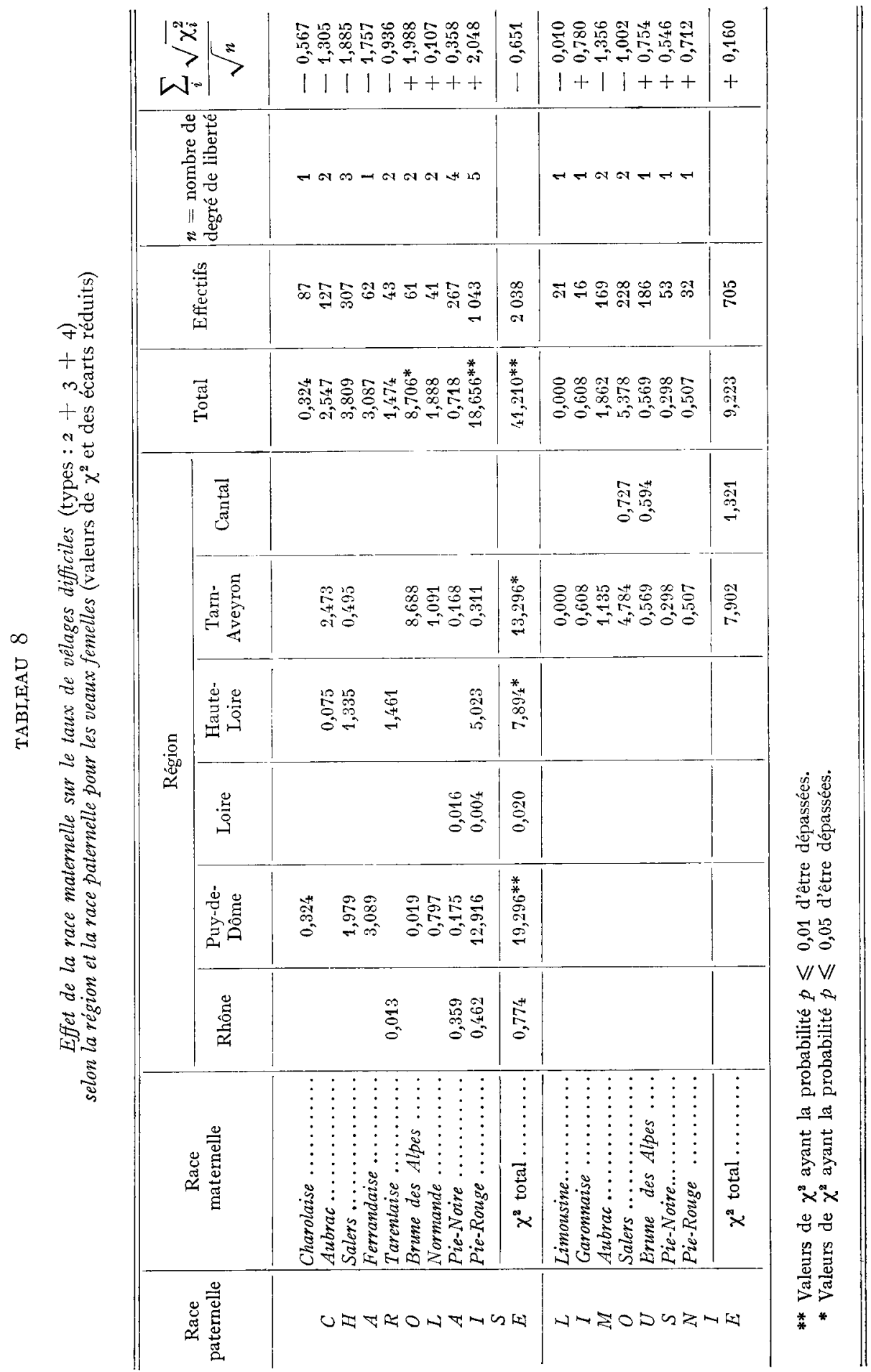




\section{TABLEAU 9}

Sélection progressive des variables permettant de discriminer les vêlages faciles ou assistés $(\mathrm{I}+2)$ des vêlages difficiles $(3+4)$ (valeurs de $\left.\Delta^{2}\right)$

A. - Avec tour de poitrine des mères (71 couples de vêlages) :

\section{Associations de variables sélectionnées}

Poids de naissance

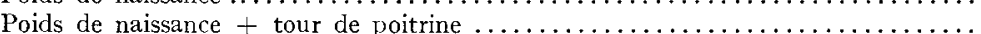

Poids de naissance $t$ tour de poitrine + poids à 75 jours $\ldots \ldots \ldots \ldots \ldots \ldots \ldots$

Poids de naissance + tour de poitrine + poids à 75 jours + prix de vente $\ldots$.
$\Delta^{2}$

0,94

1,10

1,19

1,20

B. - Sans tour de poilrine des mères (133 couples de vêlages) :

Associations de variables sćlectionnćes

Poids de naissance

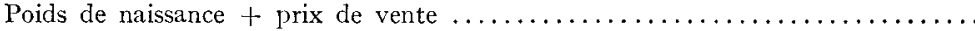

Poids de naissance + prix de vente + poids à 75 jours.

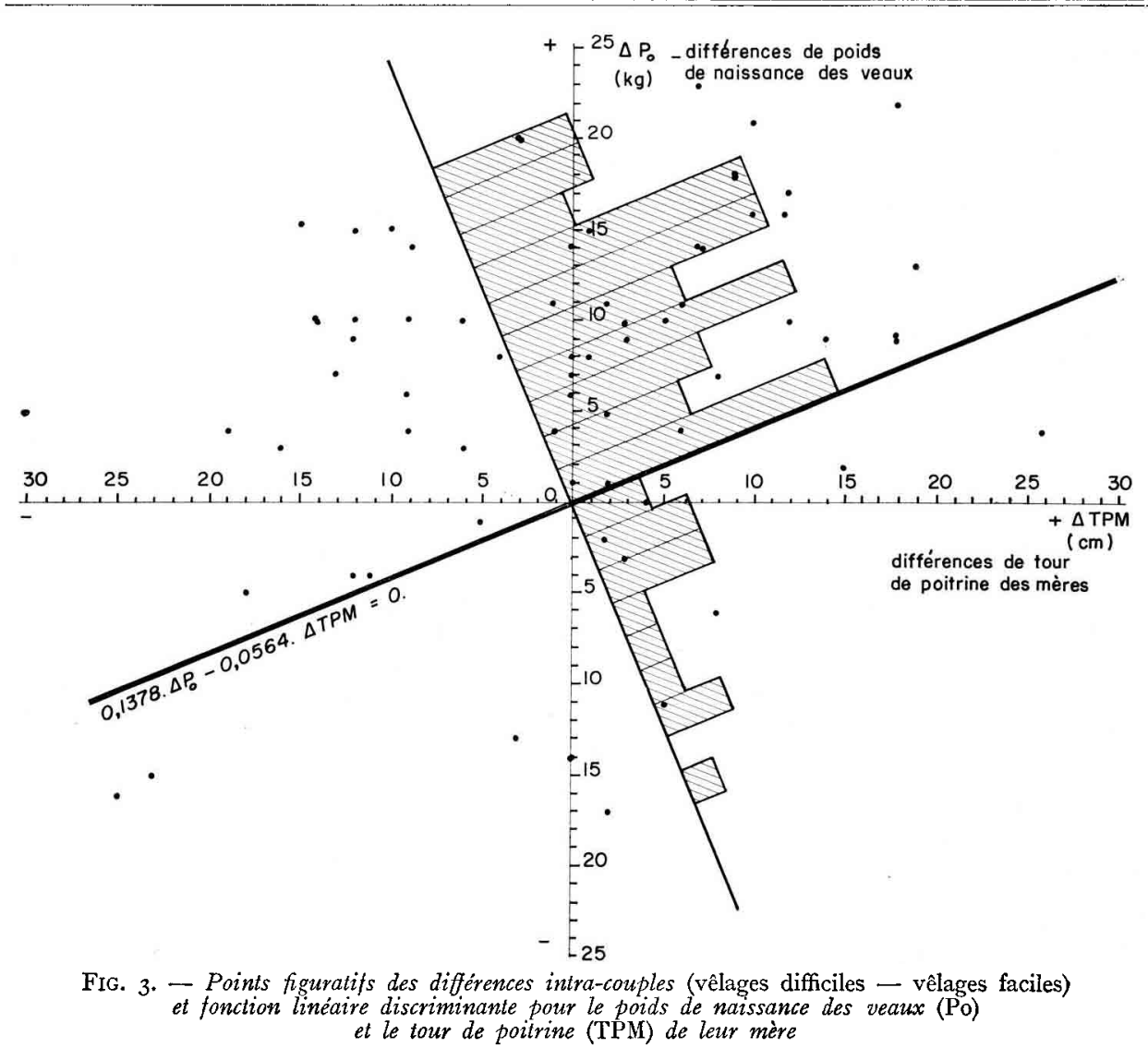


(tabl. 9). Sur ce tableau il apparaît clairement que le poids du veau à la naissance est la variable la plus liée aux difficultés de vêlage, parmi les 4 variables considérées. A poids de naissance constant, le format des mères semble avoir également une influence sur les difficultés de vêlage. Par contre après élimination de ces influences, il n'y a aucune relation entre ces difficultés et les deux autres critères considérés : poids du veau à 75 jours et prix de vente du veau surtout représentatif de son développement musculaire. A titre d'illustration, nous présentons sur la figure 3 les points figuratifs des écarts intra-couples correspondant au poids de naissance des veaux et au format des mères. Nous avons tracé la droite représentative de la fonction discriminante linéaire entre vêlages faciles et difficiles relative à ces deux variables:

$$
0, \mathrm{I} 37^{8} \cdot d_{1}-0,0564 \cdot d_{2}=0
$$

$d_{1}=$ différence intra-couple, en $\mathrm{kg}$, entre les poids de naissance des veaux;

$d_{\dot{z}}=$ différence intra-couple, en $\mathrm{cm}$, entre les tours de poitrine des mères.

La projection sur cette droite des points représentatifs des différences intracouples relatives à ces 2 variables, permet de remarquer que la valeur de cette fonction est positive dans environ $80 \mathrm{p}$. Ioo des cas. Ce chiffre situe bien l'importance de leur liaison avec les difficultés de vêlage pour des veaux de même sexe et issus des mêmes types de croisements.

\section{3. Infuence paternelle}

A partir des échantillons $n^{0} 2$ et $n^{0} 3$, il nous a été permis d'étudier l'incidence du père sur les difficultés de vêlage, selon trois caractéristiques : la race, les variations génétiques de morphologie intra-race (caractère culard) et l'individualité du géniteur.

\section{3. I. Effet de la race paternelle.}

Notre comparaison des trois principales races à viande française, porte sur les difficultés de vêlage qu'occasionnent les taureaux appartenant à ces races, en croi-

TABLEAU IO

Pourcentages de ấlages assistés, difflciles et dystociques (types $2+3+4$ ) selon le type de croisement réalisé

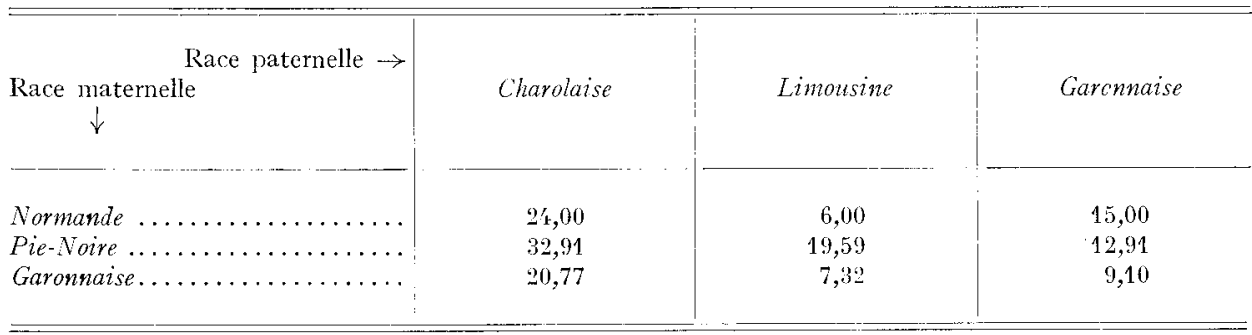

sement industriel avec des femelles adultes Normandes, Pie-Noires et Garonnaises (échantillon $\mathrm{n}^{0} 2$ ). Les taux de vêlages difficiles ou nécessitant une assistance quelconque (types: $2+3+4$ ) figurent sur le tableau Io, pour chaque type de croisement entre ces races. Nous avons comparé ensuite les races paternelles deux à deux 
en regard des difficultés de vêlage rencontrées avec chacune des trois races maternelles (tabl. II). La fréquence des vêlages assistés et difficiles, est significativement

\section{TABLEAU II}

Effet de la race paternelle sur le taux de vêlages assistés, difficiles et dystociques (types $2+3+4$ ) selon la race maternelle utilisée (valeurs de $\chi^{2}$ )

\begin{tabular}{|c|c|c|c|}
\hline $\begin{array}{r}\text { Races paternelles } \\
\text { comparćes } \rightarrow \\
\text { Races maternelles }\end{array}$ & $\begin{array}{l}\text { Charolaise } \\
\text { et } \\
\text { Limousine }\end{array}$ & $\begin{array}{l}\text { Charolaise } \\
\text { et } \\
\text { Garonnatise }\end{array}$ & $\begin{array}{l}\text { Limousine } \\
\text { et } \\
\text { Garonnaise }\end{array}$ \\
\hline $\begin{array}{l}\text { Vormande } \ldots \ldots \ldots \ldots \ldots \ldots \ldots \ldots \\
\text { Pie-Noire } \ldots \ldots \ldots \ldots \ldots \ldots \ldots \ldots \ldots \\
\text { Garonnaise } \ldots \ldots \ldots \ldots \ldots \ldots \ldots\end{array}$ & $\begin{array}{l}1 i, 9: 37^{* *} \\
4,1058^{*} \\
: 3,617\end{array}$ & $\begin{array}{l}1,280 \\
7,596^{* *} \\
5,569\end{array}$ & $\begin{array}{l}1,999 \\
1,201 \\
1,204\end{array}$ \\
\hline 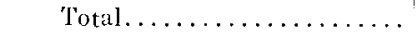 & $11,612 * * *$ & $14,1^{\prime}+5^{* *}$ & ${ }_{4}^{\prime}, y_{4}^{\prime} 0_{ \pm}^{\prime}$ \\
\hline
\end{tabular}

plus grande avec les pères Charolais (Io à $20 \mathrm{p}$. IOo) qu'avec les Limousins et les Garonnais. Par contre il n'y a pas de différences sensibles entre mâles Garonnais et mâles Limousins, pour ce critère.

\section{3. 2. Effet du caractère culard.}

Dans les deux départements où la répartition du sperme des taureaux normaux et culards a été faite au hasard (échantillon $\mathrm{n}^{0} 3$ : Rhône et Haute-Loire), nous n'observons pas de différences significatives dans la fréquence des difficultés de vêlages, selon le type de taureau utilisé (tabl. I2 et I3). Toutefois, il semble que

TABLEAU I 2

Variation du taux de vêlages assistés, difficiles et dystociques (types : $2+3+4$ ) selon le phéno'ype du père

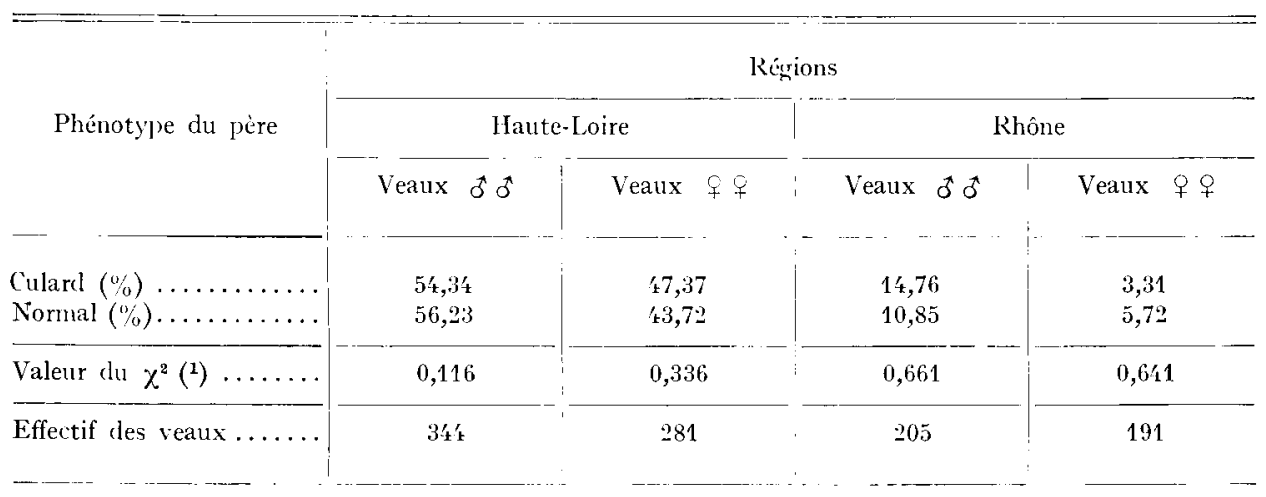

$\left.{ }^{1}\right)$ : Toutes les valeurs de $\chi^{2}$ ont une probabilité $\mathrm{P}>0,05$ d'être dépassées. 
l'emploi de taureaux culards s'accompagne d'une fréquence de vêlages difficiles et dystociques (types : 3 et 4 ) sensiblement plus élevée que celle enregistrée avec des taureaux normaux.

TABLEAU I3

Variations du pourcentage de vêlages difficiles et dystociques (types : $3+4$ ) selon le phénotype du père

\begin{tabular}{|c|c|c|c|c|}
\hline \multirow{3}{*}{ Phénotype du père } & \multicolumn{4}{|c|}{ Régions } \\
\hline & \multicolumn{2}{|c|}{ Haute-Loire } & \multicolumn{2}{|c|}{ Rhône } \\
\hline & Veaux $\sigma^{*} \sigma^{*}$ & Veaux 우우 & Veaux $0 * 0$ & Veaux 9 운 \\
\hline Culard $(\%), \ldots \ldots \ldots \ldots$ & 14,18 & 7,02 & 3,28 & 0,83 \\
\hline Normal $(\%) \ldots \ldots \ldots \ldots$ & 8,76 & 5,99 & 2,41 & 1,43 \\
\hline Valeurs de $\chi^{2}\left({ }^{1}\right) \ldots \ldots \ldots$ & 2,449 & 0,120 & 0,131 & 0,155 \\
\hline Effectif des veaux..... & $344^{\prime}$ & 281 & 205 & 191 \\
\hline
\end{tabular}

(1) : Toutes les valeurs de $\chi^{2}$ ont une probabilité $: P \geqslant 0,05$ d'être dépassées.

Le nombre réduit de veaux culards dans ces échantillons ne nous a pas permis d'analyser statistiquement la relation entre les difficultés de vêlage et le phénotype (culard ou normal) du produit.

\section{3. 3. Effet individuel des taureaux.}

Cette étude a porté sur l'ensemble de la descendance des taureaux Charolais contrôlés à l'occasion des opérations de "progeny-test " (échantillon $\mathbf{n}^{0}$ I). Du fait des variations de méthodologie du contrôle, nous avons regroupé les données en trois classes homogènes :

- classe I : centres du Rhône et de la Loire,

- classe 2 : centre du Puy-de-Dôme,

- classe 3 : centres du Tarn-Aveyron et de la Haute-Loire.

Dans chacune de ces classes, 1'influence du taureau sur les difficultés de vêlage a été déterminée à partir du coefficient de corrélation $(r)$ entre les pourcentages de vêlages assistés (types : $2+3+4$ ) pour les veaux mâles $\left(\mathrm{X}_{i}\right)$ d'une part et pour les femelles $\left(\mathrm{X}_{i}^{\prime}\right.$.) d'autre part (tabl. I4). Ces pourcentages avaient subi au préalable la

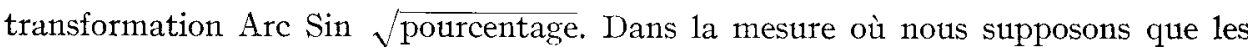
effectifs de veaux mâles et de veaux femelles issus de chaque taureau sont les mêmes (ce qui était à peu près le cas : $n$ \# 25), nous pouvons exprimer la variance due au taureau $\left(\sigma_{\iota}^{2}\right)$ par rapport à la variance totale des pourcentages de vêlages réclamant une assistance quelconque $\left(\sigma_{p}^{2}\right)$ à partir du modèle ci-dessous :

$$
\begin{aligned}
\mathrm{X}_{i}: & =\mu+t_{i}+e_{i} . \text { pour les veaux } \sigma^{\top} \sigma^{\top}, \\
\mathrm{X}_{i}^{\prime} & =\mu+t_{i}+e_{i}^{\prime} \text { pour les veaux }+q,
\end{aligned}
$$

où $\mu$ : espérance du taux de vêlages assistés pour l'ensemble des animaux. 
$t_{i}$ : effet du I eme taureau sur le taux de vêlages assistés,

$e_{i j}$ : effet aléatoire du jeme veau issu du $i$ eme taureau, sur le taux de vêlages assistés ; soit $e_{i} .=\sum_{j} e_{i j} / n$

En supposant que les $e_{i j}, e^{\prime}{ }_{i j}$ et $t_{i}$ sont indépendants, et que les variances $\sigma_{e}^{2}$ et $\sigma_{e^{\prime}}^{2}$ sont égales, nous obtenons :

$$
r=\frac{\sigma_{t}^{2}}{\sigma_{t}^{2}+\frac{\sigma_{e}^{2}}{n}}
$$

d'où

$$
\frac{\sigma_{t}^{2}}{\sigma_{t}^{2}+\sigma_{e}^{2}}=\frac{\sigma_{t}^{2}}{\sigma_{p}^{2}}=\frac{r}{r+n(\mathrm{I}-r)}
$$

L'estimée commune des coefficients de corrélations :

$$
r=+0,23 \text { (tableau : I4) correspond à } \frac{\sigma_{t}^{2}}{\sigma_{p}^{2}} \times 100=\mathrm{I}, 2 \text { p. I00 }
$$

cette valeur équivaudrait à un coefficient d'héritabilité de 0,048 .

\section{TABLEAU I4}

Coefficients de corrélation phénotypique entre les pourcentages de vêlages difficiles

\begin{tabular}{|c|c|c|c|c|c|}
\hline \multicolumn{2}{|c|}{ Variables considérées } & \multicolumn{3}{|c|}{ Classe } & \multirow[b]{2}{*}{$\begin{array}{c}\text { Corrélation } \\
\text { phénotypique } \\
r_{x y}\end{array}$} \\
\hline $\mathrm{X}$ & $\mathrm{Y}$ & $\begin{array}{c}(1) \\
\text { Rhône } \\
+ \\
\text { Loire }\end{array}$ & Puy-de-Dôme & $\begin{array}{c}\text { (3) } \\
\text { Haute-Loire } \\
+ \text { T'arn- } \\
\text { Aveyron }\end{array}$ & \\
\hline $\begin{array}{c}\text { Pourcentages } \\
\text { de vêlages assistés } \\
\left.\text { (types } 2+3+t^{\prime}\right)\end{array}$ & $\begin{array}{l}\text { Poids des veaux } \\
\dot{a} \text { la naissance }\end{array}$ & -† 0,47 & $+0,69$ & $+0,86$ & $+0,6_{\mathbf{t}}^{\prime}$ \\
\hline $\begin{array}{c}\text { Pourcentagres } \\
\text { de vêlages assistés } \\
\text { pour les veaux } \delta \precsim\end{array}$ & $\begin{array}{c}\text { Pourcentages } \\
\text { de vêlages assistés } \\
\text { pour les veaux }+?\end{array}$ & $+0,18$ & $+0,25$ & $+0,63$ & $+0,23$ \\
\hline Nombre de descendane & s considéries ........ & 30 & 28 & 15 & 73 \\
\hline
\end{tabular}

(types : $2+3+4$ ) poùr les veaux mâles et pour les veaux femelles d'une part et entre les vêlages difficiles et le poids de naissance des veaux d'un même taureau d'autre part

En procédant par la même méthode, on obtient un coefficient d'héritabilité des poids des veaux à la naissance de 0,23 . Ce chiffre est voisin de celui obtenu sur le même fichier par la décomposition classique de la variance entre demi-frères ou demi-sœurs de même père : $h^{2}=0,24$ (PoujARDIEU et VIsSAC, I968).

Quant au coefficient de corrélation entre taureaux, reliant le taux de vêlages difficiles au poids moyen des veaux à la naissance, il est de $+0,64$ (tab1. I4). I1 ressort de l'ensemble de ces résultats que l'estimation đu coefficient de corrélation génétique entre ces deux variables, est supérieure à + I pour les 73 descendances considérées. 


\section{5. - DISCUSSION}

Dans l'analyse globale des difficultés de vêlage (partie : 2), nous avons invoqué de nombreux facteurs de variations susceptibles d'entraîner des modifications du déroulement normal de la parturition. En fait, parmi les données utilisées dans notre étude, des phénomènes importants tels que : la durée de gestation, l'âge de la mère, la morphologie spécifique du bassin de celle-ci et de son veau, n'ont pu être contrôlés ou exploités. $\mathrm{L}_{\mathrm{a}}$ discussion ne portera donc que sur les points particuliers abordés dans cette étude.

\section{I. Effets liés aux caractéristiques du veau}

\section{I. I. Effet du sexe.}

Dans toutes les races maternelles analysées (sauf pour l'Aubrac dans le 'TarnAveyron), nous constatons plus de difficultés de vêlage pour les veaux mâles que pour les veaux femelles (tabl. 5). Sur l'ensemble des données regroupées indépendamment de la région, les différences de fréquence des vêlages difficiles (types : $2+3+4$ ) relatives au sexe des veaux, sont en général significatives (tabl. 5). La plupart des auteurs rapportent les mêmes écarts de fréquence entre sexes des veaux, que ce soit en race pure ou en croisement. Aurior et $a l$. (I96I), pour des veaux Charolais de race pure, trouvent 20,8 p. Ioo de vêlages assistés d'une façon quelconque avec les veaux mâles alors qu'il n'y en a que I5,3 p. Ioo avec les veaux femelles. Toujours en race pure, avec des bovins de race Pie-Noire Allemande, DREYER et SMidT (Ig66) enregistrent respectivement I3,2 p. Ioo et 5,4 p. Ioo de vêlages difficiles pour les veaux mâles et les veaux femelles. En races Pie-Noire, Pie-Rouge et de Moyenne et Haute-Belgique, HANSET (Ig66) retrouve cet effet du sexe des veaux : 54,OI p. Ioo de césarienne pour les veaux mâles au liet1 de 34,59 p. Ioo pour les veaux femelles. Ces taux très élevés de vêlages difficiles s'expliquent par le fait qu'HANSET (1966) ne considère que les vêlages produisant des veaux culards. Fin croisement, CROWLEY (I965) observe 34,o p. Ioo de vêlages dystociques pour des veaux mâles croisés Charolais $\times$ races maternelles anglaises (Shorthorn, Friesian entre autres) contre 24,8 p. Ioo pour les veaux femelles. Sur des données relatives aux mêmes types génétiques, le Vetenary Clinical Observation Unit ( 1963 ) avait enregistré 24 p. roo de vêlages dystociques pour les veaux mâles et Io p. Ioo pour les veaux femelles. HANSEN (I966) constate respectivement I6,8 p. Ioo et 9,6 p. Ioo de vêlages difficies pour les veaux mâles et femelles issus de père Charolais et de mère de race Jerseyaise. Ces fréquences de vêlages difficiles sont analogues à celles trouvées par LiNDHE (I966) : I4,54 p. I00 et 9,02 p. Ioo respectivement pour les veaux mâles et les veaux femelles, issus de femelles de race Pie-Rouge Suédoise saillies par des mâles de la même race et de races Friesian Suédoise, Rouge Danoise, Aberdeen Angus, Hereford et Charolaise.

Le poids à la naissance des veaux mâles diffère significativement de celui des veaux femelles (tabl, 4) quelle que soit la race parentale ou la région considérées. Cette différence, comme le pensent Auriol et al. (I96I), LINDHe (I966), explique en grande partie la supériorité du taux de vêlages difficiles pour les veaux mâles par 
rapport aux veaux femelles. Cependant, AURIoL et al. (I96I) remarquent que le poids moyen de naissance des veaux mâles issus de vêlages faciles sans assistance est

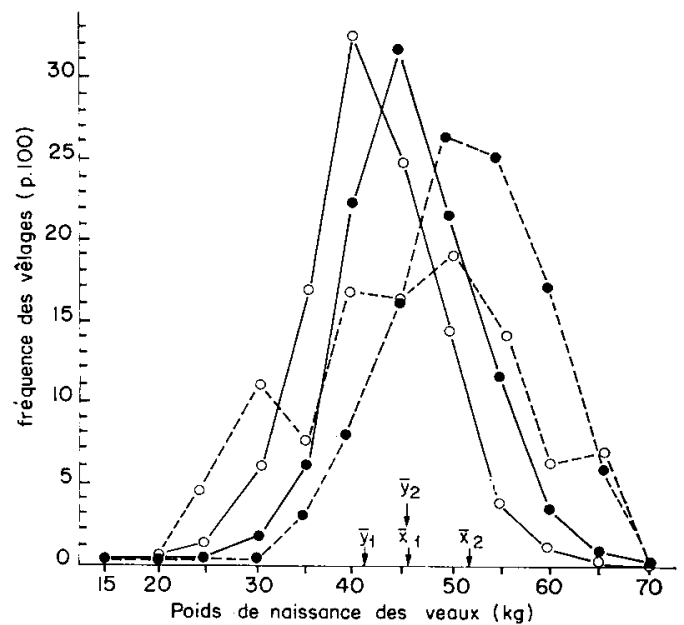

FIG. 4. - Distribution des poils te naissance des veaux croisés Charolais, suivant leur sexe et leur condition de velage

- - : Veaux ô $\hat{o}$, vêlages types : I +2 , l'oirls moyen $: \bar{x}_{1}=45,6 \mathrm{~kg}$

- - - : Veaux $\hat{0}$ ô , vêlages types : $3+4$, l'oids moyen $: \bar{x}_{2}=51,8 \mathrm{~kg}$

0 - - : Veaux $\&$ o , vêlares types : $\mathrm{I}+2$, l'oids moyen : $\mathrm{y}_{1}=4 \mathrm{I}, 6 \mathrm{~kg}$

$0 \ldots \ldots$ : Veaux + + , vêlages types $: 3+4$, l'oilds moyen $: \bar{y}_{2}=45,6 \mathrm{~kg}$

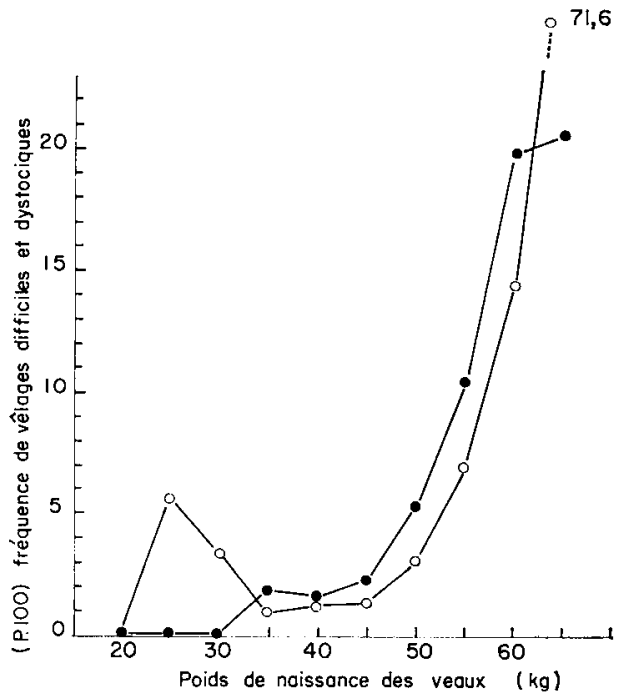

FIG. 5. - Fréquence des vêlages difficiles ou dystociques en fonction du poids de naissance des veaux selon leur sexe (veaux croisés Charolais)

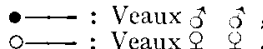

identique à celui des femelles nées d'un vêlage difficile (extraction forcée ou césarienne). Ces dernières auraient donc à poids égal une naissance plus laborieuse que les veaux mâles. AurIoL, et al. (Ig6I) attribuent cet effet spécifique du sexe des veaux 
sur le déroulement du vêlage, à la densité corporelle supérieure des veaux mâles qui entraîne, à poids égal, un encombrement moindre que celui des veaux femelles; il resterait à vérifier le fondement de cette hypothèse. Dans ce but, pour les veaux issus d'un père Charolais et d'une mère de race quelconque, nous avons établi la distribution des poids de naissance selon le sexe des vedux et leur condition de vêlage (fig. 4). Nous retrouvons les mêmes résultats qu'Aurior et al. (I96r) sur les valeurs moyennes des poids de naissance en fonction du type de vêlage. Mais, si nous prenons les veaux de même poids de naissance (fig. 5), contrairement à ce qu'affirment ces auteurs nous observons en général une fréquence de vêlages difficiles supérieure pour les veaux mâles par rapport aux veaux femelles. Ce résultat peut être interprété par la grosseur des os et le développement en largeur plus accusés, à poids égal, chez les veaux mâles. Ainsi, les seules différences de poids moyen à la naissance entre sexes ne suffisent pas à expliquer l'effet du sexe du veau sur la fréquence des vêlages difficiles. Dans le cas des races Belges produisant fréquemment des veaux culards, VANDEPLASSCHE et al. (I965) concluent également à un effet direct du sexe des veaux sur le taux de vêlages dystociques. Pour ces auteurs, les veaux mâles provoqueraient plus de vêlages difficiles du fait qu'ils sont proportionnellement plus larges que les veaux femelles.

\section{I. 2. Effet du poids à la naissance.}

Les vétérinaires praticiens tendent à considérer le volume exagéré du fotus comme cause première de leurs interventions lors des vêlages difficiles (DÉRIVAux et al., Ig64; FRIEDLI, Ig66; HANSEN, I966). D'après les résultats de notre analyse discriminante (tabl. 9) pour des veaux de même sexe, issus du même père et de femelles de la même race, le poids des veaux à la naissance semble en effet étroitement lié aux difficultés de vêlage. Dans J'essai de croisement des races anglaises avec des mâles Charolais, la Veterinary Clinical Observation Unit (I963) confirme ces résultats puisque respectivement $89,4 \mathrm{p}$. Ioo et 57,9 p. Ioo des dystocies enregistrées sont dues à une taille excessive des veaux mâles et des veaux femelles. En Belgique, VANDEPIASSCHE et al. (I965) remarquent qu'en passant de la classe de poids de naissance des veaux : $4 \mathrm{I}-45 \mathrm{~kg}$ à celle de $46-50 \mathrm{~kg}$, le taux de vêlages dystociques s'élève de I,33 p. Ioo à 2,36 p. I0o. Avec des vaches Frisonnes en race pure ou croisées avec des taureaux Charolais, REYNEKE et PENZHORN (I964) ont eux aussi montré que les poids à la naissance correspondant à des vêlages assistés par un vétérinaire, sont nettement supérieurs à ceux des veaux nés sans l'assistance vétérinaire. CROWLEY (Ig65) avec des mâles Charolais $\times$ races maternelles anglaises (Friesian, Shorthorn et autres races), LindHe (I 966) avec des femelles Pie-Ronge Suédoise croisées à des mâles de races européennes (Charolais, Aberdeen Angus, Ronge Danois, Frisons et Pie-Rouge Suédois) et Bonfert (I964) en race pure (Pie-Noire, Pie-Rouge et Simmen$t a l)$, trouvent également un accroissement du taux de vêlages difficiles avec 1'augmentation du poids de naissance des veaux. Nous avons constaté les mêmes phénomènes (fig. 5) avec les veaux de même sexe issus de pères Charolais et de mères d'une race quelconque. En définitive, il n'est pas étonnant que nous trouvions une corrélation phénotypique non négligeable $(r=+0,64)$ entre le taux de vêlages difficiles et le poids moyen des veaux à la naissance. Il est encore plus intéressant de remarquer que nous obtenons une estimation très élevée (voisine de + I) du coefficient de corrélation génétique entre ces deux variables. Ce résultat laisse donc supposer que les 
variations génétiques des difficultés de vêlage liées au père se confondent presque, dans le cas du croisement, avec celles du poids des veaux à la naissance. Or l'héritabilité de ce dernier caractère (tabl. I5) n'est pas négligeable : $h^{2}=0,23$.

\section{TABLEAU I5}

Principaux coefficients d'héritabilité $\left(\mathrm{h}^{2}\right)$

du poids de naissance des veaux, trouvés dans la bibliographie

\begin{tabular}{|c|c|c|c|}
\hline Type de bovins & Auteurs & $h^{2}$ & Race \\
\hline Bovins à viande & 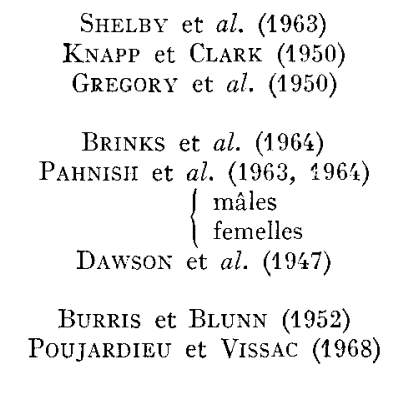 & $\begin{array}{c}0,54 \\
0,53 \\
0,45 \\
0,10 \\
0,32 \pm 0,06 \\
0,32 \pm 0,15 \\
0,14 \pm 0,11 \\
0,29 \text { (brut) } \\
0,11 \text { (corrigé) } \\
0,22 \\
0,24 \\
0,19\end{array}$ & $\begin{array}{l}\text { Hereford } \\
\text { Hereford } \\
\text { Hereford } \\
\text { Hereford } \\
\text { Hereford } \\
\text { Hereford } \\
\text { Hereford } \\
\text { Shorthorn beef } \\
\text { Shorthorn beef } \\
\text { Aberdeen Angus } \\
\text { Croisés Charolais } \\
\text { Croisés Limousin }\end{array}$ \\
\hline $\begin{array}{l}\text { Bovins laitiers } \\
\text { et mixtes }\end{array}$ & $\begin{array}{c}\text { Legault et ToucháRRY (1962) } \\
\text { (selon la méthode) } \\
\text { Plum et al. }(1965) \\
\text { EveretT et MageE (1965) }\end{array}$ & $\begin{array}{c}\begin{array}{c}0,38 \\
0,47 \\
0,41\end{array} \\
0,34 \pm 0,09 \\
0,22\end{array}$ & $\begin{array}{c}\text { de nombreuses races } \\
\text { Holstein } \\
\text { Holstein }\end{array}$ \\
\hline
\end{tabular}

De nombreux auteurs (KRASNov et PAK, I939; KNAPP et al., I940; Davis et al., I954; JoubERT et Bonsma, I959 ; BANERJEE-SChotSMAN, I964; ANDERsen et Plum, I965; Boyd et Hafs, I965, LAMpo et Willems, r965, Plum et al., ig65) ont mis en évidence des corrélations phénotypiques positives entre le poids de naissance du foetus et sa durée de vie in utero. Burris et BlunN (I952), Davis et al. (I954), Rolitins et al. (I956), BanerjeE-Scho'tsman (I964), Prabhu et al. (I966), ont également estimé le coefficient de régression du poids de naissance des veaux sur leur durée de gestation : $0,2 \mathrm{~kg} /$ jour autour de la durée de gestation moyenne (BANERJEEScho'TsMAN, I964) ; VIANNA et al. (I964) estiment respectivement à 0,243 et o,447 le coefficient de régression de la durée de gestation sur le poids à la naissance des veaux mâles et veaux femelles. Enfin, il existe une corrélation génétique élevée entre le poids de naissance des veaux et leur durée de gestation : + 0,57 (EVERETT et MAGEE, I965) + 0,37 (PLUM et al., I965). Les variations de durée de gestation influençant celles du poids de naissance, agissent donc indirectement sur les difficultés de vêlage : CROWLEY (I965), et DREYER et SMIDT (I966). Cependant, bien qu'ayant observé que les vêlages difficiles ou anormaux sont, en moyenne, associés à des durées de gestation plus longues que celles des vêlages faciles ou normaux, BANERJEE-SCHOTSMAN (I964) montre qu'indépendamment de la durée de gestation, les veaux nés de vêlages difficiles ou anormaux sont encore plus lourds en moyenne que les autres. 


\subsection{Effets liés anx caractéristiques de la mère}

\section{2. I. Effet de la race maternelle.}

Peu d'auteurs ont comparé les taux de vêlages difficiles entre plusieurs races maternelles, exploitées en race pure ou en croisement industriel avec une même race paternelle. En race pure le Midk MARkETING BOARD (I960) constate que les génisses de race Pie-Noire vêlent plus difficilement que celles de race Ayrshire (respectivement 8,8 p. Ioo et 2,5 p. Ioo de vêlages difficiles : différences significatives). Pour les mêmes races maternelles, la Veterinary Clinical Observation Unit (I963) enregistre respectivement $5 \mathrm{p}$. IOo et $4 \mathrm{p}$. Ioo de vêlages dystociques chez des femelles de tous âges. IlANcrc et al. (I964) confirment cette supériorité du taux de vêlages difficiles chez les vaches Frisonnes (4,93 p. I0o) par comparaison aux vaches Simmental (2,6I p. IOO). HANSET (I966) observe beaucoup plus de vêlages par césariennes chez les bovins des races Pie-Rouge ou de Moyenne et Haute Belgique que chez les PieNoires, les deux premières races sont, il est vrai, plus orientées vers la production d'animaux à forte musculature. Cependant, en race pure, il est difficile de distinguer la part revenant à la mère de celle attribuée au père, pour expliquer les différences entre races maternelles. En croisement, les résultats du Milk Marketing Board (I960) nous montrent que l'effet de la race maternelle enregistré ci-dessus entre les femelles Ayrshire et les femelles Pie-Noires est dû surtout à l'influence de la race paternelle. En effet, les mâles Pie-Noirs provoquent respectivement Ir,o p. Ioo et 8,8 p. Ioo de vêlages difficiles lorsqu'ils sont accouplés à des génisses de races Ayrshire et PieNoires; par contre, les mâles Ayrshire n'en produisent que 2,5 p. Ioo avec les génisses Ayrshire et 3,I p. Ioo avec les génisses Pie-Noires. Ce même organisme n'observe en outre aucune différence significative entre les fréquences de vêlages difficiles chez ces deux types de génisses lorsqu'elles sont accouplées à des mâles de races : Aberdeen Angus, Hereford (Milk Marketing Board, I960, I965) et Charolais (Milk Marketing Board, I965). A la suite d'un essai de croisement Charolais, Eidwards et al. (Ig66) confirment l'absence d'effet de la race maternelle (Ayrshire, Friesian, Shorthorn, Guernesey, Jersey) sur la fréquence des vêlages difficiles lors de croisement avec des mâles de races Charolaise, Hereford et de races identiques à celles des mères. Par contre, d'après les résultats de Lopez SAUBIDFiT et al. (Ig63) relatifs aux croisements de femelles des races à viande anglo-saxonnes (Aberdeen Angus, Hereford et Shorthorn) avec des taureaux Charolais, la race maternelle influence nettement le taux de vêlages difficiles: ce dernier varie de 4,9 p. Ioo pour les $A$ berdeen-Angus à $27 \mathrm{p}$. Ioo pour les Hereford et Io,5 p. Ioo pour les Shorthorn.

Tout comme ces derniers auteurs, nous constatons un effet significatif de la race maternelle sur le taux de vêlages difficiles. Notre étude nous a permis, d'une façon générale, de séparer les races à vocation mixte et à aire d'extension locale (Aubrac, Salers, Garonnaise) des races laitières améliorées (Brune des Alpes, Pie-Noire, PieRouge). Les vêlages apparaissent en moyenne plus difficiles (types : $2+3+4$ ) dans le second groupe que dans le premier (tabl. 7). Les autres races étudiées et notamment la race Charolaise se répartissent entre ces deux groupes. Nous remarquerons (tabl. 7 et 8) que l'effet de la race maternelle ne se manifeste vraiment que dans le Tarn, 1'Aveyron et le Puy-de-Dôme, avec les pères de race Charolaise ; cela s'explique par la composition du cheptel femelle de ces régions : celui-ci est constitué 
de races appartenant à chacun des 2 groupes ci-dessus. Enfin, l'effet de la race maternelle sur les difficultés de vêlage, reste pratiquement le même quelque soit le sexe des veaux (tabl. 7 et 8 ) ; toutefois, pour les vêlages difficiles et dystociques (types : $3+4)$, les différences entre races maternelles sont statistiquement plus nettes pour les veaux mâles que pour les veaux femelles.

L'interprétation de l'effet de la race maternelle n'est pas aisée car en dehors des considérations génétiques, les conditions d'élevage (mode de stabulation et alimentation) dans lesquelles sont entretenues les femelles des races laitières peuvent différer en moyenne de celles auxquelles sont soumises les femelles des races dites locales, à l'intérieur même d'une région. Il ne semble pas qu'il faille lier les différences de difficultés de vêlage entre races maternelles, au format général de celles-ci. Dans les deux groupes de races ci-dessus, nous trouvons à la fois des races de grand format et d'autres de format moyen. DÉrivaux et al. (I964) et FAGOT (I965) ont associé les taux élevés de vêlages difficiles dans certaines races (races Belges où le caractère culard se rencontre fréquemment) à la morphologie particulière du bassin des femelles de ces races. Nous avons vu que, d'après ces auteurs, le caractère culard entraînait une déformation du bassin caractérisé par la réduction du détroit antérieur pelvien. La manifestation externe de ce phénomène consisterait en un rétrécissement des hanches et en un élargissement des trochanters (I)ÉRIVAux et al., I964). Ces auteurs

$$
\begin{aligned}
& \text { TALIEAU I } 6 \\
& \text { Variations du rapport }=\begin{array}{l}
\text { largeur aux trochanters } \\
\text { largetur aux hanches ace la race }
\end{array}
\end{aligned}
$$

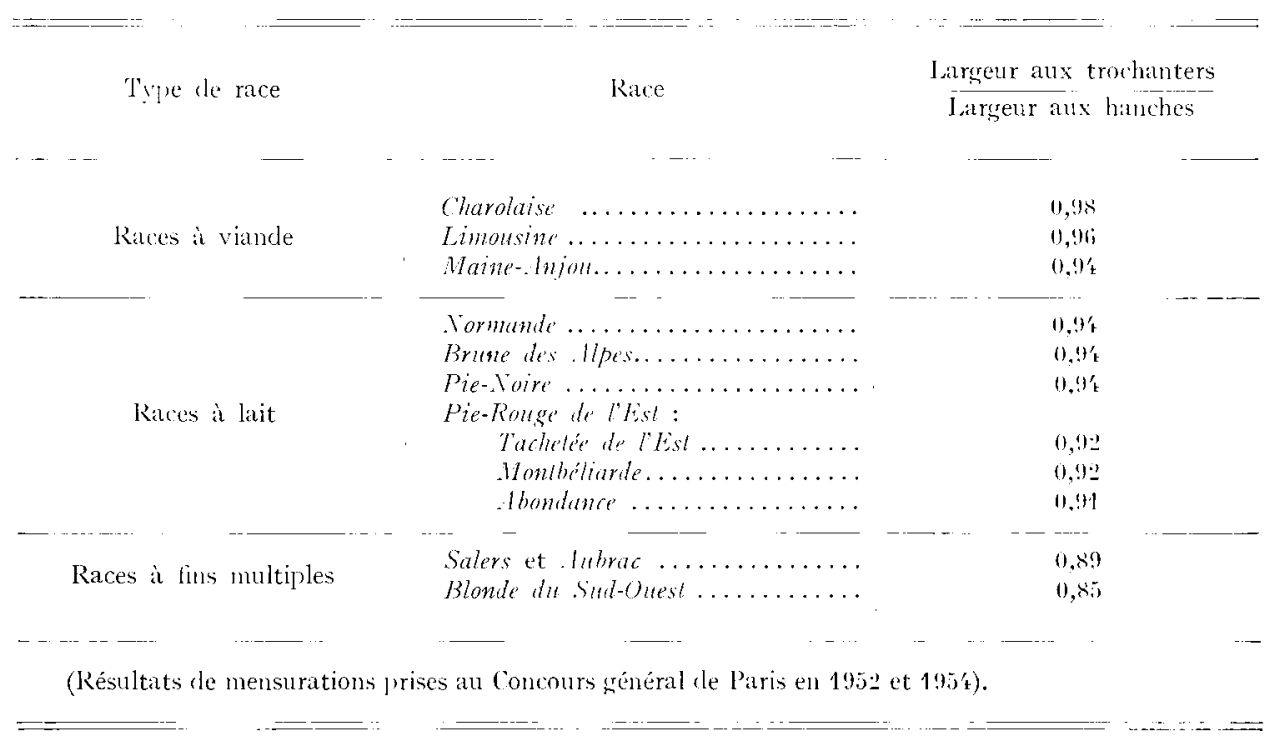

caractérisent ainsi trois types de conformation de la croupe et considèrent que le type " trapèze renversé " provoque beaucoup plus de dystocies que les deux autres. I1 est ainsi intéressant de comparer le rapport : largeur aux trochanters/largeur aux hanches, dans les races que nous avons étudiées. Celles-ci se classent dans l'ordre décroissant de ce rapport (tab1. I6). 
Malgré la taille réduite des échantillons sur lesquels ont été effectuées ces mesures, une relation apparaît nettement entre la configuration de la croupe et le classement des races en deux groupes, dans le sens indiqué par DÉRIVAux et al. (Ig64). On peut, à l'examen de ces rapports et compte tenu des enquêtes effectuées auparavant en race Charolaise (AURIor et al., I96r), s'étonner que cette race ne donne pas lieu dans notre étude à une fréquence de vêlages difficiles plus élevée. Nous pensons que cela est dû au fait que l'échantillon de femelles considéré était situé dans les zones d'extension de la race et issu de croisements d'absorption assez récents de populations locales à bassin développé par des mâles Charolais. D’après AurIoL, et al. (I96I), ce rapport largeurs aux trochanters/largeurs aux hanches varie en effet de 0,946 pour les génisses Charolaises à vêlages faciles à 0,957 pour les génisses de cette même race ayant eu une extraction forcée. Ces mêmes auteurs remarquent aussi un accroissement des difficultés de vêlage avec l'inclinaison du bassin de la mère (très caractéristique chez le bovin culard). Il convient enfin de signaler que dans ce cas limite des bovins Charolais culards pour lesquels la valeur du rapport : largeurs aux trochanters/largeurs aux hanches dépasse I (VISSAC, I968) ("trapèze renversé " de DÉRIVAUX et al. I964), tous les vêlages requièrent une assistance voire une césariectomie (Anonyma, I966). Il en est de même, semble-t-il, pour les animaux de la race $A$ shurienne présentant ce caractère (RoF CoDINA, I964). Enfin, en race Piémontaise où la fréquence des bovins culards est élevée, les difficultés apparaissent moindres (RAIMONDI, I963) et ce malgré un accroissement de durée de gestation et de poids à la naissance chez les bovins présentant ce caractère. Enfin, depuis longtemps, SAINT-CyR (I875), DEGHILAGE (IgII, cité par FAGOT, I964) et plus récemment Dickinsson (I960), DONALD (I963), Boyd et HAFs (I965), FRIEDI I (I966) ont suggéré l'existence de relations entre les difficultés de vêlages et le développement ou la conformation du bassin de la mère.

\section{2. 2. Effet du format et de l'âge de la mère.}

L'analyse discriminante entre vêlages faciles (types : $I+2$ ) et vêlages difficiles (types $3+4$ ) nous a permis de préciser l'effet de la taille de la mère sur le déroulement du vêlage. Rappelons que cette analyse a été réalisée sur des couples de vêlages consécutifs à des inséminations du même père utilisé dans la même région, avec la même race de femelle et pour des veaux de même sexe. Cet effet de la taille maternelle estimée par le tour de poitrine de la mère, se manifeste moins nettement que celui du poids des veaux à la naissance (tabl. g). Cependant l'action du format maternel n'est pas négligeable dans notre étude, et elle tend à montrer que les vaches de petite taille, dans une race donnée, vêlent plus difficilement que celles de grande taille même après avoir éliminé l'effet du poids de naissance des veaux.

Ce fait est certainement à rapprocher de la remarque de FAGOT (I964) qui signale que $30 \mathrm{p}$. Ioo des cas de césariennes ont pour cause un rétrécissement du bassin de la vache, alors que le veau reste de volume normal. D'ailleurs, VANDEPLASSCHE et al. (I965) considèrent qu'au delà d'un seuil : poids du fœetus/poids de la mère $>$ I/I2, les vêlages ont une forte chance de devenir dystociques, par incompatibilité fotomaternelle au moment de la parturition. AURIor, et al. (I96I), en comparant des tours de poitrine des mères ayant vêlé facilement, difficilement ou par extraction forcée, obtiennent des résultats moins probants quant à l'action du format de la mère sur 
les conditions de vêlage : ils trouvent peu de différences (de l'ordre de $2 \mathrm{~cm}$ ) entre les moyennes des tours de poitrine des mères de chacune des trois catégories de vêlages ci-dessus. Cela peut s'expliquer par le fait que ces auteurs n'ont pas éliminé l'effet du poids de naissance des veaux avant de procéder aux comparaisons du format maternel, contrairement à ce que nous avons fait sur nos données. En effet ces deux facteurs agissent de façon inverse sur le déroulement de la parturition : lorsque le format des mères diminue, les vêlages deviennent plus difficiles alors qu'une réduction du poids de naissance des veaux rend les vêlages plus faciles. Cependant, ces deux facteurs sont statistiquement liés, et Boyd et HAFs ( 1965$)$ ont mis en évidence des coefficients de corrélation positifs hautement significatifs entre le tour de poitrine des mères et, d'une part, le tour de poitrine $(r=+0,26)$ d'autre part, le poids de naissance des veaux $(r=+0,3 I)$.

L'effet du format maternel est vraisemblablement lié, en grande partie, à l'âge de la femelle, au moment du vêlage. Bovd et Hafs (I965) ont obtenu des coefficients de corrélation élevés entre l'âge des mères et leur tour de poitrine $(r=+0,44)$ ainsi qu'entre cet âge et le tour de poitrine des veaux $(r=+0,27)$ d'une part, et le poids de naissance des veaux $(r=+0,3 \mathrm{I})$ d'autre part. D'ailleurs, la plupart des auteurs (WithERs, I953; AURIoL et al., I96I ; DONALd, I963; Lopez-SAUBIDET et al., I963; BONFERT, I964; BALIKA, I965; DREYER et SMIDT, I966; HANSET, Ig66) indiquent tune fréquence plus élevée ( 2 fois environ) des vêlages dystociques ou difficiles chez les génisses. En effet, comme le rappellent AURIoL, et al. (I96r) soulignant qu'au delà du Io vêlage il n'y a plus de dystocies, cette différence de comportement au vêlage selon l'âge de la femelle est accentuée par l'élimination progressive des mères vêlant difficilement. En fait, dans l'effet de l'âge de la mère sur son format (donc sur les difficultés de vêlage) il faut distinguer l'incidence du nombre de vêlages de celle due plus particulièrement à l'âge de la femelle. Ainsi, dans l'enquête de REYNEKE et PENZHORN (I964), les femelles présentant des vêlages difficiles et des vêlages avec intervention du vétérinaire, avaient respectivement 7, I mois et $I 7,7$ mois de moins que les vaches vêlant facilement. Ces moyennes ont été calculées indépendamment du nombre de vêlages des femelles. Dans le cas des femelles primipares, uniquement, selon DREYER et SMIDT (I966), il semble exister une liaison positive entre l'âge et les difficultés de vêlages :

\begin{tabular}{|c|c|}
\hline Age des génisses & Taux de vêlages difficiles \\
\hline $\begin{array}{l}\text { Moins de } 24 \text { mois } \ldots \ldots \ldots \ldots \ldots \ldots \ldots \\
\text { De } 25 \text { à } 30 \text { mois } \ldots \ldots \ldots \ldots \ldots \ldots \ldots \ldots \\
\text { De } 31 \text { à } 34 \text { mois } \ldots \ldots \ldots \ldots \ldots \ldots \ldots \\
\text { Plus de } 34 \text { mois } \ldots \ldots \ldots \ldots \ldots \ldots \ldots\end{array}$ & $\begin{array}{l}12,9 \% \\
15,6 \% \\
18,8 \% \\
21,9 \%\end{array}$ \\
\hline
\end{tabular}

Ce résultat n'est en fait valable que pour des vêlages intervenant aut-delà de 20 mois, les vêlages trop précoces s'accompagnant eux aussi d'une fréquence plus élevée de difficultés. Cette incidence de l'âge de la mère, indépendante du nombre de vêlages peut présenter de l'intérêt pour certaines races comme le Charolais où les difficultés de vêlage sont fréquentes et où les primipares vêlent systématiquement à 3 ans. 


\section{3. Effets liés aux caractéristiques du père}

\section{3. I. Effet de la race paternelle.}

Dans notre étude, 1'examen du taux de vêlages difficiles selon le type de croisement pratiqué pour une race de femelle donnée, montre clairement un accroissement des difficultés de vêlage avec l'emploi de taureaux Charolais (tabl. II) par rapport aux résultats correspondant à des taureaux Garonnais et Limousins. Ce phénomène est signalé par tous les auteurs qui ont étudié le croisement avec des mâles Charolais. Pour le Veterinary Clinical Observation Unit (I963) le taux de vêlages dystociques croît de 9,7 p. Ioo pour les vaches croisées avec des mâles Hereford à I 9,8 p. Ioo pour celles inséminées avec des taureaux Charolais. EDwards et al. (Ig66) enregistrent en Grande-Bretagne des résultats équivalents, en valeur relative tout au moins: 9,52 p. Ioo de vêlages difficiles pour les croisements avec père Charolais contre 2,I2 p. Ioo pour ceux avec pères Hereford. CROWLEY (I965) enregistre également 29,6 p. Ioo de dystocies parmi les vêlages des mères Shorthorn, Pie-Noires et autres, accouplées à đes mâles Charolais. Sur ces mêmes femelles, il n'observe que I I,7 p. Ioo de dystocies avec des pères de races anglaises (Hereford, Shorthorn, Friesian et divers). HANSEN (I966) au Danemark, et LindHe (I966) en Suède, notent aussi une augmentation des difficultés de vêlages lors de 1'utilisation des mâles Charolais sur des femelles de race Jersey pour le premier auteur et de race Pie-Rouge Suédoise pour le second. Sur le continent américain, Lopez-SAUBIDET et al. (r963) observent, quant à eux, une très large variation du taux de vêlages dystociques (o à 42 p. IOO) après croisement des races à viande britannique avec des taureaux Charolais. Ces mêmes races britanniques élevées en race pure ou croisées entre elles, dans ces régions, produisent moins de 2 p. Ioo de dystocies. Comme nous l'avons déjà remarqué, ces variations du taux de vêlages dystociques dans le cas du croisement avec des mâles Charolais sont largement associées aux conditions d'alimentation des femelles pendant la gestation.

Cet effet particulier des pères Charolais semblerait résulter d'un excès de volume à la naissance des veaux croisés, fournis par ces taureaux. Pour REynEKE et PENZHORN (I964), cet écart de poids à la naissance est de 4,9 $1 \mathrm{~b}(2,22 \mathrm{~kg})$ entre les veaux Pie-Noirs purs et les croisés Charolais; Lopez-SAubide'T et al. (Ig63) trouvent 8 à Io $1 \mathrm{~b}(3,6$ à $4,5 \mathrm{~kg})$ de différence entre les veaux Angus, Hereford, Shorthorn ou leurs croisements et les veaux croisés Charolais issus de ces mêmes races maternelles. Pour EDWARDs et al. (I966) les diffétences sont du même ordre quand ils comparent les veaux croisés Charolais $\times$ Pie-Noirs ou Ayrshire aux veaux croisés Hereford $\times$ Pie-Noirs ou Ayrshire. Le Milk Marketing Board (rg64) et HANsen (I966) considèrent que l'emploi de taureau Charolais sur les femelles Jersey, accroît le poids de naissance des veaux de $70 \mathrm{p}$. Ioo environ. Il est intéressant de relier 1'accroissement de poids à la naissance des veaux croisés Charolais à l'allongement de la gestation correspondante par rapport à ce que l'on observe en race pure: de 5 à 6 jours en croisement avec les races britanniques (CROWLEY, I965; EDWARDS et al., (I966), de 2 à 3 jours avec les femelles Jersey (Hansen, I 966). Le seul exemple que nous avons recueilli, où le taureau Charolais diminue la durée de gestation des femelles qu'il saillit, est celui des croisements Charolais $\times$ Afrikander et Charolais $\times$ Bonsmara, signalé par Boyazogi U et Harwin (I966). Les femelles de ces races ont, il est vrai, une très longue durée de gestation (JoUBERT et BoNSMA, I959). 
Finalement, en dehors du volume proprement dit du fœetus, nous pouvons penser que la morphologie particulière du veau croisé Charolais (largeur de tête, des épaules et des hanches, voire hypertrophie musculaire) serait susceptible d'expliquer une partie des vêlages plus difficiles observés lors de sa naissance. La comparaison des effets des deux autres races paternelles : Limousine et Garonnaise, n'a jamais fait à notre connaissance, l'objet d'études particulières.

\section{3. 2. Effet du caractère culard chez le père.}

D'après nos résultats en croisement de première génération avec des vaches ne présentant pas le caractère culard (tabl. I2 et I3), la présence du phénotype culard chez le père des veaux n'accroît pas de façon importante la proportion de vêlages difficiles. Ce résultat est conforme à ce que nous connaissons sur la transmission du caractère culard en croisement : le poids moyen à la naissance des veaux issus de pères culards, est sensiblement supérieur à celui des veaux issus de pères ayant un phénotype normal (VISSAC, I966) mais cette différence est insuffisante pour engendrer un accroissement du taux de vêlages dystociques; de plus, les différences de développement musculaire de ces deux types de veaux n'apparaissent pas à la mise bas mais se manifestent de façon partielle au cours de la croissance suivant la phase in utéro (RAIMONDI, I965 ; VISSAC, I966). Il convient de distinguer ces études portant sur des croisements entre races ou intra-races faisant intervenir des mâles culards et des femelles normales de l'enquête effectuée sur la race de Moyenne et Haute Belgique (HANSET ig66) où des femelles sont sélectionnées sur leur conformation bouchère. Cet auteur a observé des différences de difficultés de vêlage entre taureaux : les mâles présentant les fréquences de dystocies les plus élevées dans leur descendance, donnent également un pourcentage plus élevé de veaux culards.

\section{3. 3. Effet du génotype paternel.}

Le coefficient d'héritabilité que nous avons obtenu pour le taux de vêlages difficiles $(0,05)$ doit être interprété avec prudence : il ne correspond qu'à l'effet direct du père sur les veaux qu'il engendre et non à son action sur les troubles de parturition de ses filles. Nous avons souligné la liaison étroite qui existait entre cette variation génétique et celle du poids de naissance des veaux. Or la réduction du poids de naissance et l'accroissement de la croissance post-natale des veaux croisés paraissent difficiles à réaliser simultanément par sélection en race Charolaise. D'une part, la phase de croissance concernée est très courte (3-4 mois), d'autre part le coefficient d'héritabilité de la croissance pendant cette phase est très faible $(0,05)$, celui du poids à la naissance étant proportionnellement plus élevé $(0,25)$ et la corrélation génétique entre ces deux variables étant très forte (PoujARdieu et Vissac, I968).

Peu d'auteurs ont étudié statistiquement les différences de difficultés de vêlages entre descendances de mâles. Cependant, VAN DiÉreN (I963), EDwards (I966), DREYER et SMIDT (I966), et LINDHE (I966) signalent des différences notables de fréquences de vêlages difficiles ou dystociques entre descendances de taureaux.

L'exemple de DREYER et SMIDT (I965) renforce l'intérêt que nous devons porter sur l'effet héréditaire du père au niveau des difficultés de vêlage. Ces auteurs observent en effet d'importantes différences d'une part entre les taux de vêlages difficiles engendrés par les taureaux (de 23,5 p. Ioo de vêlages difficiles à 3,8 p. Ioo selon le

Annales de Zootechne. - I968. 
mâle) et, d'autre part, entre les taux de vêlages difficiles enregistrés sur les groupes de demi-sours paternelles issues de taureaux d'insémination artificielle (de $\Upsilon_{7}, 7$ p. Ioo à 5,5 p. I00). L'accouplement du taureau ayant donné le taux le plus élevé de vêlages difficiles (23,5 p. IOO) avec respectivement les filles des deux taureaux donnant des femelles ayant les vêlages les plus difficiles ( 77,7 p. I oo et I2,6 p. IOO), conduit à l'enregistrement respectif de 53,8 p. Ioo et 63,6 p. Ioo de vêlages difficiles parmi ces accouplements. Ce résultat spectaculaire témoigne des possibilités et des risques d'une sélection vis-à-vis des conditions de vêlages.

\section{6. - CONCLUSIONS}

Les difficultés de vêlage par incompatibilité morphologique foeto-maternelle sont, en définitive, influencées par un grand nombre de facteurs. L'action de ces derniers ne semble pas obéir à un modèle additif, ce qui est logique, les troubles de parturition étant liés à un phénomène de seuil; ainsi l'effet de la race maternelle apparaît plus nettement lors des vêlages de veaux mâles qui requièrent plus fréquemment une assistance. De même les difficultés liées à l'emploi de mâles culards semblent plus fréquentes dans les populations où le cheptel femelle comporte des animaux présentant ce caractère.

Dans le cas du croisement de première génération que nous avons étudié ici, les incidences paternelles et maternelles sur les difficultés de vêlage doivent être séparées et sélectionnées indépendamment. Les différences entre femelles apparaissent d'abord quand on compare les races locales aux races laitières améliorées plus sensibles aux troubles de mise-bas. Le remplacement progressif des premières par les secondes dans beaucoup de régions où l'on pratique le croisement industriel est à ce sujet préoccupant. Ces différences raciales semblent liées à la morphologie spécifique du bassin plus qu'au format des femelles. Cette morphologie peut être appréciée par le rapport des largeurs aux trochanters et aux hanches lié lui-même aux dimensions de l'ouverture pelvienne. L'étude du bassin des femelles culardes, considérées à cet égard comme un cas limite, a permis d'apprécier cette liaison (DÉrivaux et al., I964). Nous savons aussi que des mesures directes de cette ouverture peuvent être pratiquées (WILTBANK, I96I; BENDAVID, I960), et donner lieu à une sélection des races maternelles dont l'efficacité maximum pourrait être obtenue par une sélection massale des mâles de ces races destinées au testage (MENissien, I967).

Les variations de fréquence des difficultés de vêlage entre races ou familles paternelles apparaissent nettement : on sait que le croisement Charolais donne souvent lieu à des difficultés de ce type, les variations génétiques de fréquence de ces difficultés entre descendances de taureaux appartenant à cette race sont faibles (5 p. Ioo) et liées très étroitement à celles du poids de naissance. L'étude des paramètres de la variation génétique de croissance des veaux croisés permet à ce sujet de conclure qu'une augmentation de cette dernière dans la phase post-natale et indépendamment de la croissance in utéro, sera difficile à obtenir par sélection des mâles Charolais destinés au croisement. Peut-être assistons-nous là à une limite économique de la sélection pour la croissance pondérale? Peut-être aussi est-il possible de sélectionner 
des souches mâles pour le croisement industriel dont la morphologie serait à format équivalent plus adaptée au vêlage : types longilignes à os fins?

En tout état de cause, les combinaisons entre races maternelles et races ou familles paternelles du point de vue des difficultés de vêlage méritent une étude particulière, le seuil critique n'est probablement pas le même d'une race maternelle à une autre pour un génotype paternel donné ; le format des mères, la morphologie de leur bassin ainsi que leur effet spécifique sur la croissance du veau in utéro varient d'une race maternelle à l'autre, or le dernier effet notamment est assez mal connu.

Ręu pour publication en janvier 1968.

\section{REMERCIEMENTS}

Nous tenons à remercier ici les organisations d'élevage (Centres d'insémination et Syndicats de contrôle de croissance) (jui, en collectant l'information que nous avons exploitée, ont permis la réalisation de ce travail. Les données relatives aux comparaisons entre races paternelles nous ont par ailleurs été fournies par M. BoNHounE que nous tenons à associer à ce travail.

Enfin, nous remercions plus particulièrement M. VISSAC, de nous avoir guidés tout au long de ce travail at dans l'interprétation de nos résultats.

\section{SUMMARY}

\section{SOME FACTORS INFLUENCING DIFFICULT CALVING}

IN INDUSTRIAL CROSS BREEDING

Industrial cross-breeding is more and nore used for improving the muscular growth of young steers. It implies matings of bulls with a high rate muscular growth with cows of smaller size and lower compactness, which often induce difficult calvings.

The factors responsible for dystocic calvings linked with a dam foctus incompatibility act either on the opening of the Pelvian ductus of females or on the size of the foetus. But dystocic calvings can have various complex origins (fig. I).

The occurence of difficult calvings was studied on 7500 offspring of Charolaise and Limousin breeds used for cross-breeding from 1960 to 1965 . These calvings have been tabularized for centres and breeds in table $\mathrm{I}$. To investigate upon the influence of the sire s breed, we have taken into account calves sired by Chraolais, Limousin, Garonnais bulls mated to adult dams of Normande, Pie-Noire and Garonnaise breeds exclusively (table 2 ).

The influence of the "double muscle " character of the sire was comparatively investigated on double-muscled and normal Charolais sires mated with adult range dairy breeds of dams (table 3 )

Male embryos induce many more difficult calvings than the female, whatever [the dam's breed (tables 4, 5 and 6). This can be due to the sex differences in weight at birth (table 4). But this explanation is not sufficient, for an analysis of the frecluency distribution of weights at birth, depending upon sex and type of calving (fig. I), showed that there were more difficuit calvings with male calves than with female, for a given weight at birth (fig. 2). This can be due to the conformation and carcass development of male calves.

We have shown that for calves of the same sex, bon from the same sire, and identical dam's breed in the same district, difficult calvings were most related to weight at birth (table 9). The dan's size had only a secondary influence.

From a general standpoint, this study enabled us to distinguish local breeds of dams serving a double purpose (Aubrac, Salcrs, Garonnaise) from improved dairy breeds (Brune des Alpes; Pie Noire, Pie Rouge), the former's calvings being less difficult than the latter's (table 7 ). All other breeds studied were pooled within the 2 groups. The differences between dam breeds were irrespective of the sex of calves (table 7 and 8). The classification of breeds based on the ratio : width at trochanters/width at hindquarters (table $\mathbf{I}_{5}$ ) shows that a relationship exists between conformation of pelvis and difficult calvings. 
Matings of a given breed of dams with Charolais sires increased the proportion of difficult calvings (tables ro and II) compared with Limousin and Garonnais. The typical morphology of Charolais-crossed calves can partly account for this increase. The double-muscled character in the sire did not significantly increase the proportion of difficult calvings (tables $\mathbf{I}_{2}$ and $\mathrm{I}_{3}$ ) in industrial cross-breeding.

The direct influence of the bull on difficult calvings of his progeny was estimated with Charolais bulls, from the correlation coefficients between the percent attended calvings for male of female calves (table I4). The heritability coefficient was thus $h^{2}=0.05$.

Lastly, the correlation coefficients between the rates of difficult calvings and the average weight at birth of calves from the same sire were high (table I4) : genetic correlation $r=+$ r.20.

Therefore, the inter-sire variations in difficult calvings in industrial cross-breeding, are dosely related to weights at birth. Since the heritability coefficient of this criterion is higher with Charolaise breed than that of the post parturient growth will hardly be possible without a corresponding increase in dystocic calvings. Moreover, substituting narrow-hinded dams from dairy breeds to wide-hinded dams from local breeds shall induce more frequent difficult calvings. These results must be taken into account when choosing the cross-breeding of the first generation.

\section{RÉFÉRENCES BIBLIOGRAPHIQULS}

Andersen H., Plum M., I965. Gestation length and birth weight in cattle and buffaloes. A review. $J$. Dairy Sci., 48, I 224-1 235 .

Anonyme, rg66. Document de travail sur les recherches françaises concernant le caractère culard. Europ. Assoc. Anim. Prod., Comm. Anim. Genet., 9h Int. Cong\%. Anim. Prod. (Edinburgh), 6 pp.

Auriol P., Dumont B.-L., Lefebve J., Duplay J. M., ig6r. Caractéristiques générales des vaches Charolaises et croissance de leurs produits. Hrance-Elevage Charolais, t; $28 \mathrm{pp}$.

J3ALIKA S., 1965. Data on heavy parturition of cattle. Allaltenyésatés, 14, 229-234.

BARone R., 1966 . Anatomie comparée des mamitères domesiques. Tome 1. - Ostéologie, 81 I pp., Lab. d'anat., Éc. nat. vét. de Lyon.

Banerjee-Schotsmann I., 1964. A study concerning gestation period in callle. A biomelrical contribution. These Utrecht, $167 \mathrm{pp}$.

BEn DaviD B., I 960 . Contributi ed osservazioni pratiche. Sulla distocia causate da un feto relativamente grande nella vacca Frisona israeliana. Clinica vet., Milano, 83, 412-419.

BEN DAVID B., I964. Obstetric measurement of the pelvis in Israeli-Friesian heifers during pregnancy as compared to those of their cowes at the time of parturition. Refuah vet., 21, 49-45.

BONFERT A., ig64. Kontrolle der Kalbungen von Besamungsbullen. Tiersitchter, 16, 885-886.

Boyd L. T., HaFs H. D., rg65. Body size of calves from Holstein darns and sired by Holsiein or $4 n$ gus bulls. J. Dair Sci., 48, I $236-1240$.

Boyazoglu J. G., Harwin G. 0., ig66. Le Charolais dans les croisements en élevage extensif dans les pays chauds. Charolais, $1966,4,57-65$.

Brinks J. S., Clark R. T., KiefFer N. M., URick J. J., I964. Estimates of genetic, environmental and phenotypic parameters in range Herelord females. J. Anim. Sci., 23, 7 I I-7 6 .

Burris M. J., BuUNN C. T., I952. Some factors affecting gestation length and birth weight of beef cattle. J. Anim. Sci., 11, 34-41.

Cabrini E. J. J., Cavandoli H. H., 1964. Cruzas con Charolés en Marcos Juarez. Boln. téc. Estac. exp. agropec., Marcos Juarez, 1, I-34 (in Anim. Breed. Abstr., 34, 26).

ClegG M. T., in Coes H. H., Cupps P. T., i959. Reproduction in domestic animals, vol. I,509-538, Academic Press, New York and Londen.

Crowley J. P., I965. The effect of Charolais bulls on calving performance. Ir. J. agric. Res., 4, $205-213$.

Davis H. P., PLum M., Brost B., I954. Studies of herd management records. 2. Relation of gestation length to birth weight of Holstein calves of both sexes at various calvings. J. Dairy Sci., 3\%, $162-166$.

Dawson W. M., Phillips R. W., Black W. H., 1947. Birth weight as a criterion of selection in beef cattle. J. Anim. Sci., 6, 246-257.

Derivaux J., r957. Obstétrique vétérinaire. 392 pp., Vigot frères, Paris et Desoer S. A., Liège.

Derivaux J., Fagot V., Huet R., 1964. Le problème des dystocies. Essais de pelvimétrie. Ann. Méd. vét., 108, 335-365.

Dickinson A. G., ig60. Some genetic implications of matemal effects. An hypothesis of mammalian growth. J. Agric. Sci., 54, 378-390.

Dieten S. W. J. Van, I963. Morlalité des veaur lors du port à terme en race Meuse-Rhin-Yssel (en hollandais). Thèse vét. Utrecht, $188 \mathrm{pp}$. 
Donalu H. P., I963. Perinatal deaths among calves in a crossbred dairy herd. Anim, Prod., 5, 87-95.

Dreyer D., SMIDT Dr., 1966. Welche Bedeutung haben Schwergeburten und Kälberverluste für die züchterische Beurteilung von Besamungsbullen? Tier ï̈hler, 18, 528-529.

EuWards J., i966. Production de viande réalisée à partir du Charolais utilisé en croisement sur les races laitières en (irande-I3retagne. Charolais, 4, 35-4 I.

Emwards J., Jobst D., Hodges J., Leybure M., O’Connor L. K., Mac Donald A., Smitil G. F Woon P., I 666 . The Charolais Report. Milk Mikty Bd, $47 \mathrm{pp}$.

Evenett R. W., MAi:eE W. T., 1965. Matemal ability and genetic ability of birth weight and gestation length. J. Dairy Sci., 58, 957-96r.

Fariot V., Ig6+. La vraie conformation de boucherie en rapport avec les possibilités de vêlage. Zoolechnia $13,3-17$.

FAco'T V., ro65. Dystocies et pelvimétrie. Zoootechnia, 14, 60-64.

FriedLI U., I 966 . Statistische Untersuchungen über Schwergeburten beim Rind. Schaeiz. lantac. Mh. 44, $275^{-277}$.

Gregory K. F., Blexi C. T., Baker M. L., I050. A study of some of the factors influencing the birth and weaning weights of beer calves. 1 . inim. Sci., 9, 338-3-16.

Grunsell C. S., Messervy A., Pearson II., Wilson M. R., ig6.3. The Charolais survey. Vet. Rec., 75 , I I5I.

HaynoxD I., 1955. Progress in the physiologl of farm animals, Vol. 2, 395-740, Butterworths scie.ntific publications, London.

IIANSEN I. H., ig66. The incidence of dystocia and postparturient disorders in Jersey cattle after cross breeding with Charnlais bulls. Br. vet. J., 122, 273-278.

IANSET A., ig66. Le problème de l'hypertrophie musculaire ou caractire "Culard " dans la race bovine de Moyeme et Haute Belgique. Europ. Issoc. Anim. Prod. Comm. Anim. Genet., gth Int. Congr. Anim. Prod., (Edinburgh), 21 pp.

Ilancic D., Penavix V., Seinovic I., Ig64. Comparative examination of the frequency of some puerperal diseases in the black-white (Friesian) and Simental cows. Veterinaria Sari., 13, 175-183.

Jafar S. M., Chapman A. B., Casida L. E., 1950. Causes of variation in length of gestation in dairy cattle J. Anim. Sci., 9, 593-601.

Jor bFRI I'. M., Boxism J. C., I959. Cestation of cattle in the sub-tropics, with special reference to the birth weight of calves. S. Ifr. J. Agric. Sci., 2, 2 1 $5^{-2} 30$.

Jocber' D. M., Hammond J., I958. A crossbreeding experiment with cattle, with special reference to the matemal effects in Soulh-Deonn $\times$ Dexter crosses. J. Agric. Sci, 51, 325-34I.

KNAPP B. Jr., CLARK R. T., I950. Revised estimates of heritability of economic characters in beef cattle. I. Anim. Sci, 9, $582-587$.

KNAPP B. J., LAMbiRT W. W., Black W. H., Ig4o. Fiators inlluencing length of gestation and birth weight in cattle. I. agric. Res., 61, $227-285$.

Krasyov K. E., PAK D. N., I939. An attempt of testing tagil bulls by the birth weight of their progeny Anim. Breed. Abstr, 7 , 108 .

Lampo P., Willevs $\Lambda .$, i 965 . Causes de variations du poids de naissance des veaux (en flamand). Vlaams Diergeneesk. Tiilschr., 34, 79-84.

Legacte C., Totcirgerry R. W., i 962. Heritability of birth weight and its relationship with production in dairy cattle. J. Dairv Sci, 45, 1226-г 233 .

LiNDIE 13, Ig66. Crossbreeding for beef with Swedish Red anl while calle. 93 pp. Part I and II. Thise.

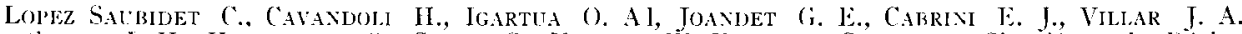
Sivori I. II., Hernandez O., Covas G., Kinier W. F., I963. Cruzas con Charolés en la Région P'ampeant. Boln. lec. Eslac. Exp. Agroper. Balcarce, I. N. T. A. 6, i-74.

Mrissier F., ig67. (données non publices).

Milk Marketixg Bonk1, i965. The Charolais trials. Rep. Brecal. Prod.Org. Milk Mlktg Bd, Thames Jitton Surrey, 15, $43-47$.

Milk Marketing Bonkd, ig64. The Charolais Class i $\mathrm{C}$ " trials. Rep. Breed. Prod. Org., Milk Mktg Bd, Thames Ditton Surrey, 14, ro3-108.

Milk Marketix; BonRd, igon. The incidence of difficult calving in fyrhire and firiesian heifers. in Rep. Breed. Prod. Org., Milk Mktor Bd, Thames Ditton Surrey, 10, g8-99.

l'ahnish O. F., Robertson R. L., Taylor R. J., l3rinks J. S., Clark R. T., Roubicek C. I3., i936. Genetics growth traits in range cattle. J. Inim. Sci., 22, 8ㄷ (abstr.).

Painish O. F., Robertion R. L., Taylor R. l., Brinks J. S.., Clark R. T., Roubicek C. B., 1964. Genetic analysis of economic traits measured in range-raised Hereforls at preweaning and weaning ages. f. Anim. Sci., 23, $562-568$.

Ply M., ANDersex H., Swicier L. A., ig65. Iteritability estimates of gestation length and birth weigh in Holstein-Friesian cattle and their use in selection indexes. J. Dairy Sci., 48, 1672-1675.

Poljarideu B., Vissac B., 5968. Ftude biométrique de la croissance et de la valeur bouchère de veaux croisés Charolais et I.imousins. I. Paramètres génétiques et phénotypiques Anns Zoolech. (sous presse). 


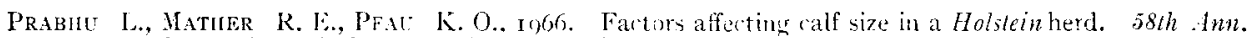
Mect. Am. Soc. Anim. Sci. (Rutger), l. Inim. Sci., 25, S79 (abitr.).

Ramonne R., 1963 . Risultati di una prova di allevannento di bovine l'iemmesi del tipo "della coscas" Annali Sper. agr.. 17, $471-4 i 99$.

Ranioxin R., 1965. Résultats des essais de croisoments entre des taureaux Piémonlais de bourherie et des vaches de races laitières. World h'm. Anim. Prod., l (Special issue), sy-c)1.

RFYMFNT R. $\Lambda .$, I 962 . Observations on homogeneity of covariance matrices in paleontologic biometry. Biontirics, 18, I-1 I.

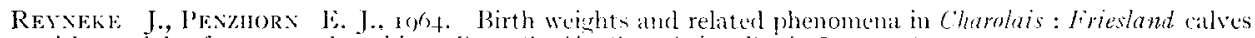
with special reference to dystolit. Proc. S. .lfi. Soc. Inim. Prod., 3, 160-160.

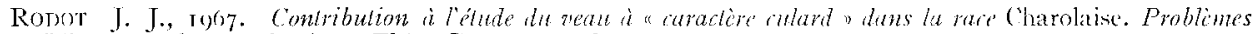

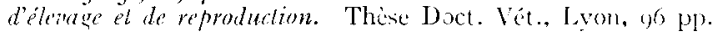

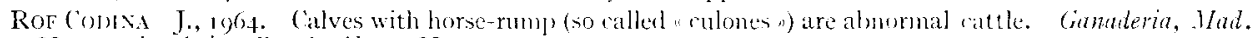
22, 2.. in Inim. Breet. Abstr., 32, 1953.

Roldins W. C., laben R. C., Mest S. W., I 956 . (iestation length in an inbred fersey herd. J. Dairy Sci., 39, I $578-1593$.

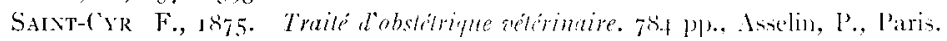

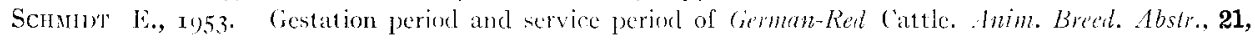
712.

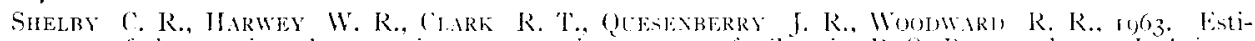
mates of phenotypic and grenotypic paraneters in ten years of miles city R. O. P. steer data. J. Ham.

Sci.22, 34(3)-35.3.

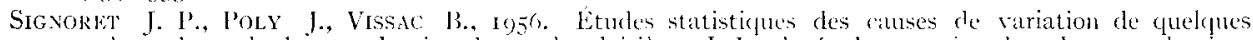
parametres du cycle de reproduction des vaches laitieres. I. Lat duréc de teestation dans les races bovines

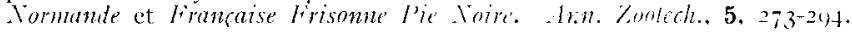

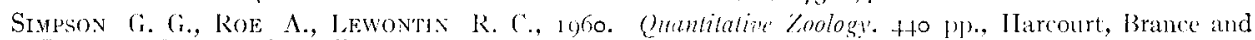
Company, New lork Burlingame.

Stockkialswer li., I95. Bericht iber die Forschungen auf dem Gebiet der Hohemiehzucht von 1945 bis 1954. Zïhlungstiunde, 26, $237-257$.

Swanson E. W., Hinton Si. A., Iong. Effere of seriously restricted growth upon lactation. J. Jainy Sci., 47, 267-27?.

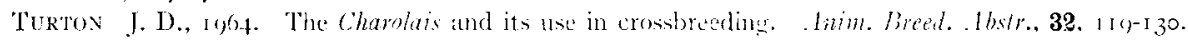

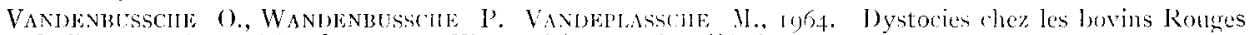
de l'()uest, Filamand (en flamand). Vlaams liergeneesh. Tildsder. 33, 20,5-215.

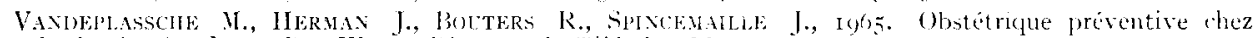
les bovins (en lamand). Vlaams liergeneesh. Tijilsch., 34, ion-172.

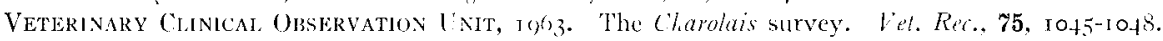

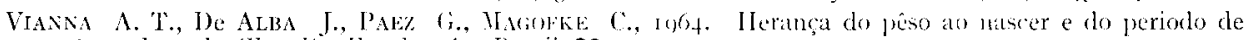
gest içao do wado Charolés. Estudos téc, Brasil, 26.

Vissac 13., 1962. I'remieres recherches francaises sur le curactere culard. C. R. Jonmées d'Eules F. E. Z. (Viennc), i 5 pp.

Vissac 13., r966. Communication personnelle.

VIssac 13., Ig68. Etude du carartére culard. Il. Incidence du caractére culard sur la morphologie générale des bovins. Ann. Zootech. (à paraitre).

Vissac B., Frebling J., Faucon A., 1965. Statistiques grénérales sur la production de veaux de boucherie en croisement industriel dans le Centre et le Sud-Ouent de la France. Bull. Lech. Ini., Ingrs Serv's agric. 204, $889-939$.

Wagner R., r965. Sélection progressive de variables utilisant la statistique $D^{2}$ de Mahalanobis. Application à la détermination de la meilleure fonction discriminante séparant deux populations de semis de vigne. Ann. Amélior. Pl. 15, 159-181.

Wiltbank J. N., ig6r. Save more alves at birth. . Veb. Expt. Sla. Quaterly. Summer ed., i pp.

Wrtuers F. W., 1953. Mortality rates and discase incidence in calves in relation to feeding, managemen! and other envirommental factors. P'art VI. Brit. arel. /. 109, iz2. 